\title{
Escalation in conflict games: on beliefs and selection
}

\author{
Kai A. Konrad ${ }^{1} \cdot$ Florian Morath ${ }^{2}$
}

Received: 8 January 2019 / Revised: 18 August 2019 / Accepted: 21 October 2019 /

Published online: 19 November 2019

(c) The Author(s) 2019

\begin{abstract}
We study learning and selection and their implications for possible effort escalation in a simple game of dynamic property rights conflict: a multi-stage contest with random resolve. Accounting for the empirically well-documented heterogeneity of behavioral motives of players in such games turns the interaction into a dynamic game of incomplete information. In contrast to the standard benchmark with complete information, the perfect Bayesian equilibrium features social projection and type-dependent escalation of efforts caused by learning. A corresponding experimental setup provides evidence for type heterogeneity, for belief formation and updating, for self-selection and for escalation of efforts in later stages.
\end{abstract}

Keywords Dynamic conflict · Lottery contest · Heterogeneity $\cdot$ Incomplete information $\cdot$ Uncertainty $\cdot$ Escalation $\cdot$ Beliefs $\cdot$ Selection $\cdot$ Learning $\cdot$ Social projection · Experiment

JEL Classification C90 · D72 $\cdot$ D74 $\cdot$ D83

A preliminary version of this paper circulated with a slightly different title, "Escalation in dynamic conflict: On beliefs and selection" We benefited from comments at conferences, workshops and seminars at Beijing, Berlin, Chapman, Cornell, EPCS Rome, Frankfurt, Maastricht, Munich, Norwich, Rotterdam, Vanderbilt, Vienna, and York. The usual caveat applies.

Electronic supplementary material The online version of this article (https://doi.org/10.1007/s 1068 3-019-09630-1) contains supplementary material, which is available to authorized users.

Florian Morath

florian.morath@uibk.ac.at

Kai A. Konrad

kai.konrad@tax.mpg.de

1 Max Planck Institute for Tax Law and Public Finance, Marstallplatz 1, 80539 Munich, Germany

2 University of Innsbruck, Universitaetsstr. 15, 6020 Innsbruck, Austria 


\section{Introduction}

Substantial research has been devoted to the study of conflict, described as an adversarial interaction between players who expend efforts and try to achieve mutually exclusive goals. ${ }^{1}$ We study dynamic properties of such conflict if the contestants take part in a sequence of pairwise contests, have incomplete information about their opponent's type and learn from the interactions about the composition of the population of potential future opponents.

Extensive experimental work on conflict confronts theory predictions with subjects' behavior in the laboratory and has uncovered systematic behavioral departures from the complete information benchmark models. One main interpretation of the findings is that the individuals who interact in conflict games-in the laboratory and elsewhere-follow motives beyond the maximization of monetary payoffs and that these motives are not uniform across individuals. Whereas monetary incentives are typically common knowledge in experimental setups, these other (intrinsic) motivations are not; this turns the interaction into a game with incomplete information. Individuals may know their own preferences but have to form beliefs about the types of opponents they interact with. If players interact repeatedly with other players from the same population (experimental session), they may learn about other players' types, update their beliefs about the type of future opponents and adjust their behavior accordingly, which may lead to an escalation or de-escalation of contest effort.

The importance of unobserved heterogeneity in individual motivations for behavior in strategic interactions such as conflict games has three main implications, which establish the research agenda of this paper. First, a suitable benchmark model of conflict games needs to incorporate incomplete information about individuals' 'preference types' such as their intrinsic motivation to win. To allow for learning about the population of opponents, the model needs to be dynamic. Second, observed adjustments of behavior in the experimental data should be contrasted with the equilibrium prediction of the dynamic game, which can be structurally different from the complete information benchmark. Third, individual heterogeneity and learning can cause self-selection if the likelihood of future interactions is not fully exogenous such as in models of dynamic conflict that consist of several "battles".

Our framework adds a simple dynamic structure to a generic model of distributional conflict. ${ }^{2}$ In the multi-stage game considered, each contest stage takes the form of a standard two-player Tullock contest, with the only modification that the

\footnotetext{
1 Generic examples are patent races (Loury 1979; Lee and Wilde 1980; Harris and Vickers 1987), procurement (Fullerton and McAfee 1999; Che and Gale 2003), military campaigns, political competition and lobbying (Ellingsen 1991; Baye et al. 1993; Klumpp and Polborn 2006; Meirowitz 2008). Comprehensive surveys of the theory of conflict are Konrad (2009) and Garfinkel and Skaperdas (2012).

2 For the variant of the Tullock contest that we consider it is well documented that behavior in the laboratory deviates systematically from the standard theory prediction based on symmetric players who maximize monetary payoffs. The survey on contest experiments by Sheremeta (2013) discusses various sources for the observed heterogeneity in efforts, which are based on unobservable individual characteristics.
} 
prize is awarded with some (exogenous) probability only. Otherwise the game moves to the next contest stage, the players are re-matched in pairs and choose new efforts. ${ }^{3}$ The conflict game is set up such that effects of belief updating and self-selection based on unobserved preference heterogeneity can be isolated in the experimental data. Ignoring preference heterogeneity, a standard theory with complete information (without population uncertainty) would predict behavior to be identical across all stages of conflict. This is mainly due to the assumption of an exogenous continuation probability which, hence, plays a key role for the identification strategy by removing the strategic links between the stages, except possibly for belief updating. ${ }^{4}$

Unobserved heterogeneity in characteristics such as the intrinsic motivation to win and uncertainty about the composition of the population of opponents introduce dynamics of conflict behavior caused by belief formation and updating. In line with a theory of social projection, we show that a player's own preference type partially shapes her beliefs about other participants. ${ }^{5}$ The different player types start with different beliefs about the population of opponents. Learning about the opponents' propensity to exert effort has different strategic implications for players who are strongly or weakly intrinsically motivated. Intuitively, learning that a majority of opponents have a weak intrinsic motivation (choose low effort) reduces the incentive to exert effort for strongly motivated participants, as they can ensure a high win probability at lower costs. However, the same signal about the opponents increases the incentive to exert effort for weakly motivated participants, similar to an encouragement effect. This logic is closely related to strategic considerations in other contest applications and is a consequence of the usual non-monotonicity of best reply functions.

In the closely corresponding baseline experimental treatment (BASE), a monetary prize is awarded based on a Tullock function with probability $1 / 3$ in a given stage, which would end the game. In each stage reached, the players are randomly

\footnotetext{
${ }^{3}$ For the sake of illustration we may think of lobbying campaigns by interest groups where policy reform implementation is an uncertain event. When players lobby for different policy outcomes, the game may end at a given stage because a final policy decision has been made. However, a new policy-maker or a new government may be elected before the lobbying efforts pay off, making old efforts obsolete and opening up for a new round of efforts. Ben-Bassat's (2011) study highlights the relevance of multiple decision makers in the adoption process of reform and the factors that make adoption more likely.

${ }^{4}$ As a side effect, random continuation allows to observe dynamics of effort choices in situations where a contestant neither won nor lost the previous contest. Related to this, there is a discussion about a potential "reinforcement effect" of winning the previous round on efforts in the next round; see Sheremeta (2013) for a survey of experimental results and Gauriot and Page (2019) for a clean identification based on field data from tennis. In our design, adjustments of efforts can be attributed to belief updating since the 'neutral' outcome (prize not yet allocated) arises exogenously and thus cannot be correlated with unobserved preference heterogeneity, in contrast to a victory in the previous round.

${ }^{5}$ The idea that others might be like ourselves and that players give much (actually too much) weight to this hypothesis is studied in social psychology and led to the theory of social projection. In their overview of the 'false consensus effect', Marks and Miller (1987) survey theories and document empirical evidence on social projection, according to which individuals think that their own choices and judgements are more frequent. Social projection may occur for beliefs, attributes, behaviors, and other personal characteristics, including objectives (see Krispenz et al. 2016, p. 867; Kawada et al. 2004).
} 
matched in pairs and, apart from choosing contest expenditures, have to state their beliefs about the opponent's effort. If the prize has not been awarded after 5 stages, the game ends without prize allocation. We find considerable evidence for nonincentivized heterogeneity among players which-together with the implied updating of beliefs in later stages - also explains dynamic adjustments of contest efforts across the contest stages. Unobserved preference heterogeneity and belief formation about potential opponents are seemingly a driver of the observed effort escalation and deescalation; not allowing for this type of information asymmetries would yield theory predictions that are structurally different from the experimental findings.

As a main experimental variation (the EXIT treatment), we consider a variant of the game that allows for self-selection-an aspect which is crucial in most dynamic conflict games where participation at later stages is not fully exogenous. ${ }^{6}$ To emphasize the implications of self-selection in the presence of unobserved preference heterogeneity, we extend the multi-stage contest by an explicit continuation decision after the first contest encounter (to be made in case the prize has not yet been awarded). The outside option is chosen such that equilibrium behavior based on monetary payoff maximization does not change, that is, it is lower than the equilibrium expected continuation payoff. The experimental results, however, confirm our theory prediction that unobserved preference heterogeneity and updating about the population of future opponents can cause self-selection of certain types into continuing conflict and result in an escalation of efforts in later contest stages.

Our paper is the first to develop and test a theory of sequential contests in which conflict behavior may be driven by intrinsic (behavioral) motives, in which players cannot observe the intensity of the motives of their competitors, and in which they are uncertain about the environment described by the distribution of types of possible competitors. While the dynamics of conflict caused by population uncertainty and belief updating have not been studied, some elements of this theory relate to results that have been developed in the theory of all-pay contests with incomplete information in a purely static context. Malueg and Yates (2004) analyze the static contest between two players whose prize valuations are drawn from a commonly known binary distribution. Even though their information assumption differs from the one that emerges from social projection in our dynamic framework, their results are structurally similar to stage 1 of our game. Fey (2008), Ryvkin (2010), Wasser (2013a, b) and Einy et al. (2015) study existence of Bayesian equilibrium in the static incomplete information Tullock contest. ${ }^{7}$ The results by Einy et al. (2015) are closest to our existence results, as they allow players to have private information about the state of nature.

Broad empirical evidence on conflict behavior suggests an intrinsic motivation to win, leading to a mismatch between a complete information benchmark and the

\footnotetext{
6 The selection incentives are related to Wärneryd's (2012) analysis of entry in a game in which some players know the value of the prize and others do not. His framework is static and players are ex ante asymmetric with respect to what they know.

7 For an experimental comparison of lottery contests under complete and incomplete information see Brookins and Ryvkin (2014). Their two-player setup with a known probability distribution of the effort costs exhibits no significant differences in effort choices as compared to the complete information case.
} 
experimental results. Moreover, contest efforts often exhibit dynamics that do not square with the standard theory intuition. ${ }^{8}$ Other findings suggest that self-selection based on unobserved heterogeneity of contestants may explain deviations from the complete information benchmark model. In line with this, Fu et al. (2013) show that players sometimes engage in costly messages prior to a lottery contest and explore the role of incomplete information as the rationale for this behavior. Herbst (2016) finds selection effects which she explains by players' differences in a 'joy of winning'. Herbst et al. (2015) consider unobserved behavioral heterogeneity of players in the context of free-riding in fighting alliances and the endogenous versus exogenous formation of such alliances. They also find that players make inference from past actions of their co-players, and weak players exploit strong players if both types enter into the same fighting alliance. Strong players understand this and tend to self-select: rather than joining the fighting alliance with a player who is likely to be weakly motivated, they prefer to become stand-alone fighters. In our paper, random re-matching after each interaction avoids that players can make inference on the behavioral type of their specific co-player. However, the players learn about the nature of the overall population. This population learning turns out to be sufficient for an adjustment of their behavior and for whether to continue to participate in the conflict game or quit.

Another dimension of learning dynamics in experimental contests concerns the extent to which feedback is provided, with mixed evidence so far. In a setting with fixed matching of participants, Fallucchi et al. (2013) find that information about the opponent's choice has opposite effects on effort levels in probabilistic and deterministic contests. Mago et al. (2016) consider four-player contests with fixed matching and find no effect of information about others' effort on average efforts but dynamic adjustments of efforts which reduce effort heterogeneity (the latter is in line with our theory; the former may arise from the predicted countervailing adjustments of different player types). Keeping the set of choices observed at each stage constant and eliminating learning about the specific opponents and hence strategic signaling by design, our approach addresses the idea that different types of players may hold systematically different beliefs, which can lead to different adjustments of beliefs and efforts when learning about the population of potential opponents.

Our paper also relates to a methodological discussion of the benchmark choice in laboratory experiments. If players understand that their co-players do not play the money-guided Nash equilibrium action, this should trigger a different optimal reply, even for strictly money-oriented players. Fudenberg and Levine (1997) find evidence in experimental contexts that actual co-players' behavior may induce learning and may cause players to optimize against this observed behavior. Konrad et al. (2014) report similar findings in the context of monopoly pricing and consumer boycott. Camerer and Weigelt (1988) study experimental behavior in a finite lending game with reputation building. In their context, players have incomplete information

\footnotetext{
${ }^{8}$ See, for instance, the recent experimental results by Deck and Sheremeta (2012, 2019), Mago et al. (2013) and Gelder and Kovenock (2017). Dechenaux et al. (2015) provide a comprehensive survey on contest experiments.
} 
about other players' monetary incentives by experimental design of the game. Our approach combines elements of these approaches. We do not induce heterogeneity in incentives or incomplete information about these incentives. We rather draw on experimental evidence that finds players' heterogeneity along an important ('behavioral') dimension and acknowledge that subjects have incomplete information about the non-monetary payoff components of their opponents. ${ }^{9}$

The role of population uncertainty, self-projection and Bayesian learning may be important in various contexts beyond contest applications. Ample evidence has shown that many players who interact in a laboratory environment have motives in addition to the extrinsic monetary incentives provided. ${ }^{10}$ Since players cannot really know the distribution of types in a subject pool when taking part in an experiment, a well-reasoned choice requires players who enter a laboratory session to form a belief about the composition of the population of subjects from which the co-players are drawn. It may be appealing early on to make Bayesian inference from one's own type and update this belief from interaction to interaction. With an increasing number of observations of others' choices the importance of a player's own type for the beliefs about the opponents' types may fade.

\section{Theoretical framework}

\subsection{Model}

We consider a framework in which conflict about a prize takes place in up to $n$ stages, each described as a Tullock contest. The players differ in their prize valuation and there is uncertainty about the probability distribution of types. The theory framework allows for learning about the true type distribution but, by construction, removes strategic aspects of how own effort choices are interpreted by others. One variant of the analysis allows for players' selection by including the possibility to exit the multi-stage game.

Players, actions, and timing Let $I$ be an infinitely large set of players. The game has up to $n$ stages but may end before reaching the terminal stage. In any given nonterminal stage $s<n$, if this stage is reached, each player $i$ is randomly matched with one other player $-i$. Players $i$ and $-i$ simultaneously choose efforts $x_{i, s} \in[\underline{x}, \bar{x}]$ and

\footnotetext{
${ }^{9}$ Heterogeneity in extrinsic motivations would just add another layer of heterogeneity, without eliminating the importance of heterogeneity in intrinsic motivations. For an example see Herbst et al. (2015) who show that players self-select into groups based on both, extrinsic (incentivized) and intrinsic (nonincentivized) motivations.

10 These include tastes for procedural fairness, for efficiency, for consistent behavior, feelings of altruism or spite, a preference for honesty, a quest for recognition, considerations of self-respect and self-image, status considerations, equity concerns, and others. It has been shown that a given population of subjects in the laboratory is heterogeneous in the intensity of these motives. For instance, Kerschbamer et al. (2017) develop tests to identify subjects' social preferences (selfish, efficiency loving, spiteful, inequality averse, inequality loving) and find considerable heterogeneity among subjects.
} 
$x_{-i, s} \in[\underline{x}, \bar{x}]$, where $0<\underline{x}<\bar{x}$. This leads to one of three outcomes, described from the point of view of player $i$. In the first outcome $i$ wins a prize and reaches no further stage (that is, the game ends for $i$ ). In the second outcome player $-i$ wins this prize; again, $i$ reaches no further stage (the game ends for $i$ ). In the third outcome none of the two players wins the prize but the game continues for $i$ who enters stage $s+1$. This third outcome emerges with probability $1-q$. This probability is exogenously given and does not depend on $x_{i, s}$ or $x_{-i, s}$. The other two outcomes emerge with probabilities $q p_{i, s}\left(x_{i, s}, x_{-i, s}\right)$ and $q\left(1-p_{i, s}\left(x_{i, s}, x_{-i, s}\right)\right)$. As will become clear below, the assumption of an exogenous continuation probability $1-q$ is the key assumption that allows to isolate selection effects in the experimental data.

The function $p_{i, s}\left(x_{i, s}, x_{-i, s}\right)$ describes the win probability of player $i$ in stage $s$, conditional on the prize being awarded in this stage. This conditional probability is a function of the player's own effort and the opponent $-i$ 's effort at this stage. We assume that this function is given by the Tullock (1980) contest success function:

$$
p_{i, s}\left(x_{i, s}, x_{-i, s}\right)=\frac{x_{i, s}}{x_{i, s}+x_{-i, s}}
$$

for all stages $s=1, \ldots, n .^{11}$ The function $p_{i, s}\left(x_{i, s}, x_{-i, s}\right)$ is continuous, strictly increasing and concave in player $i$ 's own effort and strictly decreasing in the effort of the opponent $-i$ of this stage. ${ }^{12}$

If the prize is not allocated in stage $s<n$ such that player $i$ enters into stage $s+1$, the players are randomly re-matched. Hence, the identity of the opponent typically changes between stages, as the set $I$ of players is infinitely large. We denote by $i$ a given player (with unchanged identity over all stages that are reached) and by $-i$ the opponent assigned to player $i$ in a given stage. In stage $s+1$, player $i$ and the new opponent $-i$ choose efforts $x_{i, s+1} \in[\underline{x}, \bar{x}]$ and $x_{-i, s+1} \in[\underline{x}, \bar{x}]$ and the stage contest resolves according to the same rules as in stage $s$. This continues until the game ends for $i$ because one of the players wins the prize, or until the terminal stage $s=n$ is reached. Interaction at the terminal stage $n$ follows the same rules as in previous stages, with one difference: should none of the two players win at stage $n$, the game ends and no prize is awarded.

Payoffs Payoffs consist of the prize value if the player wins, minus the own effort costs that are 'all pay': Players $i$ and $-i$ pay the cost of their own efforts $x_{i, s}$ and $x_{-i, s}$. By normalization, these costs are equal to $x_{i, s}$ and $x_{-i, s}$. They occur independent of whether or not a player wins at stage $s$, or whether the prize is awarded in this stage at all. There is no discounting, so effort costs add up for the different stages.

\footnotetext{
11 Tullock (1980) introduced (1) in the area of rent-seeking contests. Hirshleifer and Riley (1992), Baye and Hoppe (2003) and Jia (2008) describe microeconomic underpinnings for this function. Skaperdas (1996) and Clark and Riis (1998) offer axiomatic justifications. The function has been used in many areas (see, Konrad 2009, pp. 43-44).

${ }^{12}$ By assuming $x_{i, s} \in[\underline{x}, \bar{x}]$ with $0<\underline{x}$, the denominator in (1) is strictly positive, which avoids the discontinuity problem at $\left(x_{i, s}, x_{-i, s}\right)=(0,0)$ in the standard setup (where $p_{i, s}(0,0)$ needs to be defined separately). The lower bound $x$ on efforts should be thought of as being positive but very close to zero. The upper bound $\bar{x}$ should be thought of as being very large such that a choice of $x_{i, s}=\bar{x}$ generates a negative payoff and is dominated by, for instance, $x_{i, s}=\underline{x}$.
} 
Player $i$ values winning the prize by $v_{i}>0$, which is private information. This fact and the probability model that describes the random process behind the assignment of valuations is common knowledge and formalized below. Player $i$ learns her own prize valuation $v_{i}$ prior to the first effort choice in stage 1 . We assume that a player keeps this valuation of winning throughout all stages of the game. Players may differ in their valuation of the prize: one share of players has a valuation $v_{L}$, the other share of players has a valuation $v_{H}>v_{L}{ }^{13}$ We sometimes call a player with valuation $v_{L}$ a 'weak' player and a player with valuation $v_{H}$ a 'strong' player. As there is no discounting, a player has the same benefit from winning the prize if she wins at stage $s$ as if she wins at stage $s+k$.

Altogether, player $i$ 's payoff is $v_{i}-\sum_{k=1}^{k=s} x_{i, k}$ if $i$ wins the prize at stage $s,-\Sigma_{k=1}^{k=s} x_{i, k}$ if $-i$ wins the prize at stage $s$, and $-\sum_{k=1}^{k=n} x_{i, k}$ if the prize is not allocated at any stage $s=1, \ldots, n$.

Population uncertainty and information structure The prize valuation $v_{i}$ is assigned to player $i$ in a random process that has two layers of randomness. First, there are two possible states of the world that may prevail. These states $\bar{\omega}$ and $\underline{\omega}$ differ in the probability distribution from which the players' valuations are drawn. All players start with common prior beliefs $\sigma_{i}(\omega)$ about the probability that the world is in state $\omega \in\{\bar{\omega}, \underline{\omega}\}$ and attach a probability of $1 / 2$ to each of the two possible states.

Second, nature draws the type of each player $i \in I$ independently from the same given probability distribution, which depends on the state of the world. Specifically, in state $\omega$ of the world, player $i$ is assigned valuation $v_{H}$ with probability $\pi_{\omega}$, and is assigned valuation $v_{L}$ with the complementary probability $1-\pi_{\omega}$; we assume that

$$
\pi_{\bar{\omega}}=\frac{1}{2}+d \text { and } \pi_{\underline{\omega}}=\frac{1}{2}-d, \text { where } d \in\left(0, \frac{1}{2}\right) .
$$

Hence, the state of the world characterizes the share of high types, $\pi_{\omega}$, and the share of low types, $1-\pi_{\omega}$, in the population; the share of high types is larger in state $\bar{\omega}$ than in state $\underline{\omega}$. For $d>0$ the player's own type as well as the experienced opponents' efforts affect the players' updating about the probability for the world to be in state $\bar{\omega}$ or $\underline{\omega}$.

Beliefs At the beginning of stage 1, each player $i \in I$ learns her valuation $v_{i}$, which $i$ keeps throughout the game. As $v_{i}$ is a random draw from the true probability

\footnotetext{
13 As will become clear below, the intuition for the main theory predictions does not rely on the assumption of heterogeneity in the specific domain of prize valuations or a single dimension of preference heterogeneity. In the experimental framework with monetary prizes, one possible motivation behind the assumption of different prize valuations is that some players may, in addition to the monetary prize, attribute a non-monetary value to the event of winning. We may, for instance, think of $v_{L}$ as the monetary value of the prize and $v_{H}-v_{L}$ as the monetary equivalent of a high type's non-monetary benefit from winning. Such a "joy of winning" has been discussed extensively and analyzed experimentally (see Herbst 2016 for a survey of this discussion, and an experiment that focuses on measuring the joy of winning). The assumed difference in valuations can also have other reasons, such as differences in altruism or status considerations.
} 
distribution (which is one of two possible ones), this makes $i$ 's own valuation not only important for the payoff from winning but also for the beliefs about other players' valuations: player $i$ uses Bayes' rule to update her belief about the true state of the world, which is then used to determine her beliefs about the composition of the population from which the opponent $-i$ is drawn. In stages $s \geq 2$ (if reached), the beliefs also depend on the history of opponents' efforts in previous stages $1, \ldots, s-1$.

Formally, in stage $s$ the population is composed of players with different prize valuations $v_{i}$ and different histories of observed efforts of previous opponents $-i$; the vector

$$
\mathbf{h}_{i, s} \equiv\left(v_{i}, x_{-i, 1}, \ldots, x_{-i, s-1}\right)
$$

describes all relevant information about a player's genuine type (prize valuation) and experience type (history of opponents' effort choices) at the beginning of stage $s$. Somewhat loosely we refer to $\mathbf{h}_{i, s} \in H_{s}$ as $i$ 's 'type' in stage $s$ where $H_{s}$ denotes the set of types in stage $s$. For a player $i$ of type $\mathbf{h}_{i, s}$ to be teamed up with player $j$ of type $\mathbf{h}_{j, s}$, the probability beliefs are characterized by cumulative distribution functions $F_{\mathbf{h}_{i, s}}\left(\mathbf{h}_{j, s}\right)$. Atoms in these distributions will be denoted by $\rho_{\mathbf{h}_{i, s}}\left(\mathbf{h}_{j, s}\right)$; they measure the probability which a player $i$ with valuation $v_{i}$ and experienced opponents' effort $\left(x_{-i, 1}, \ldots, x_{-i, s-1}\right)$ attributes to the event that her newly matched opponent $-i=j$ in stage $s$ has a prize valuation $v_{j}$ and experienced previous opponents with expenditures $\left(x_{-j, 1}, \ldots, x_{-j, s-1}\right)$.

\subsection{Benchmark: no uncertainty about the state of the world}

Before analyzing the model with population uncertainty and Bayesian updating, we consider a benchmark case for which the true state $\omega \in\{\bar{\omega}, \underline{\omega}\}$ is common knowledge and player types are independently drawn from the respective distribution in which $\pi_{\omega}$ is the known share of players with valuation $v_{H}$.

Proposition 1 Suppose that there is common knowledge about the share $\pi_{\omega}$ of highvaluation players and denote the equilibrium efforts by $\breve{x}_{v_{H}}$ and $\breve{x}_{v_{L}}$ for players with valuation $v_{H}$ and $v_{L}$, respectively. Then, $\breve{x}_{v_{H}}$ and $\breve{x}_{v_{L}}$ are constant across all stages $s$ that are reached. All player types expect that their rival's effort is, on average,

$$
E_{v_{H}}\left(x_{-i, S}\right)=E_{v_{L}}\left(x_{-i, s}\right)=\pi_{\omega} \breve{x}_{v_{H}}+\left(1-\pi_{\omega}\right) \breve{x}_{v_{L}} .
$$

The proofs of this and all subsequent propositions are in Appendix 1. In the benchmark of a known distribution of types, a player cannot learn from her own type or other players' effort about future opponents' types. Thus, each of the stages can be seen as independent; the dynamic game can be interpreted as a sequence of 
completely independent static games. ${ }^{14}$ An interior equilibrium $\left(\breve{x}_{v_{H}}, \breve{x}_{v_{L}}\right) \in(\underline{x}, \bar{x})^{2}$ at a given stage is described by the first-order conditions

$$
\breve{x}_{v_{H}}=\pi_{\omega} \frac{q v_{H}}{4}+\left(1-\pi_{\omega}\right) \frac{\breve{x}_{v_{L}} \breve{x}_{v_{H}}}{\left(\breve{x}_{v_{H}}+\breve{x}_{v_{L}}\right)^{2}} q v_{H}
$$

and

$$
\breve{x}_{v_{L}}=\pi_{\omega} \frac{\breve{x}_{v_{H}} \breve{x}_{v_{L}}}{\left(\breve{x}_{v_{L}}+\breve{x}_{v_{H}}\right)^{2}} q v_{L}+\left(1-\pi_{\omega}\right) \frac{q v_{L}}{4} .
$$

The equilibrium levels of a player are precisely the same in each stage $s$ and the players' expectations of the rival's effort are independent of the own player type.

Special parametric cases have been solved explicitly in the literature. If $\pi_{\omega}=1 / 2$, the first-order conditions become

$$
\breve{x}_{v_{i}}=\left[\frac{1}{8}+\frac{\breve{x}_{v_{H}} \breve{x}_{v_{L}}}{2\left(\breve{x}_{v_{L}}+\breve{x}_{v_{H}}\right)^{2}}\right] q v_{i} \text { for } i \in\{H, L\}
$$

[as in Malueg and Yates (2004)]. For the case of symmetric players with $v_{H}=v_{L}=v$, this solution reduces to $\breve{x}_{v}=q v / 4$, which corresponds to the result obtained by Tullock (1980).

\subsection{Perfect Bayesian equilibrium with learning}

This section contains the theory results for the framework with uncertainty about the distribution of types. We show existence of a perfect Bayesian equilibrium of the dynamic game and offer a partial characterization of the equilibrium efforts.

Proposition 2 A perfect Bayesian equilibrium in pure strategies exists.

In each stage, players form beliefs about the composition of the set of players conditional on their own valuation and the behavior of players they have previously been matched with. The equilibrium beliefs at each stage are characterized by finite sets of mass points $\rho_{\mathbf{h}_{i, s}}\left(\mathbf{h}_{-i, s}\right)$. Based on these the players maximize their expected payoff in the contest of the respective stage. Compactness and continuity properties of the optimal choices allow us to apply Brouwer's fixed point theorem to conclude

\footnotetext{
14 While the equilibrium effort choices are unchanged in each period, a dynamic aspect of the game is that a player's expected continuation payoff and probability to eventually win the prize are decreasing in $s$.
} 
that this class of problems has a fixed point that characterizes an equilibrium of the static Bayesian game at each stage.

The linkage between stages is via belief updating about the composition of the set of possible opponents. Random re-matching of players at each stage and the size of the set of possible opponents become important here, causing that player $i$ 's effort choice at a given stage only affects the future beliefs of a finite number of other players that form a set of zero probability mass. From a single player's perspective this turns the problem into a sequence of structurally independent Bayesian games.

Stage 1 properties At the beginning of stage 1, the players' beliefs depend on their own valuation only, that is, types $\mathbf{h}_{i, 1} \in\left\{v_{H}, v_{L}\right\}$.

Lemma 1 In stage $s=1$, players with valuation $v_{H}$ and $v_{L}$, respectively, believe that the share of high-valuation players in the population is given by

$$
\rho_{v_{H}}\left(v_{H}\right)=\frac{1}{2}+2 d^{2} \text { and } \rho_{v_{L}}\left(v_{H}\right)=\frac{1}{2}-2 d^{2} .
$$

Using straightforward Bayesian updating, the beliefs in (7) are derived in two steps. First, type $\mathbf{h}_{i, 1}$ updates her beliefs $\sigma_{\mathbf{h}_{i, 1}}(\omega)$ about the probability that the true state of the world is $\omega \in\{\bar{\omega}, \underline{\omega}\}$. The beliefs about the share of high types in (7) follow directly from $\sigma_{\mathbf{h}_{i, 1}}(\omega)$ and Bayes' rule. As (7) shows, each player believes that the state of the world is more likely in which the player's own type is more likely, and thus believes that it is more likely to face an opponent of the same type. Lemma 1 can explain if players of different types form different beliefs about their opponent's type and, hence, effort in the first stage. The next proposition characterizes explicitly the stage 1 equilibrium efforts $x_{\mathbf{h}_{i, 1}}^{*}$ and the players' expectations $E_{\mathbf{h}_{i, 1}}\left(x_{-i, 1}\right)$ about their opponent's effort.

Proposition 3 Denote the equilibrium efforts in stage 1 by $x_{v_{H}^{*}}^{*}$ and $x_{v_{L}}^{*}$ for players with valuations $v_{H}$ and $v_{L}$. If $\left(x_{v_{H}}^{*}, x_{v_{L}}^{*}\right) \in(\underline{x}, \bar{x})^{2}$, these efforts are equal to

$$
x_{v_{H}}^{*}=\rho_{v_{H}}\left(v_{H}\right) \frac{q v_{H}}{4}+\left(1-\rho_{v_{H}}\left(v_{H}\right)\right) \frac{q v_{H}^{2} v_{L}}{\left(v_{H}+v_{L}\right)^{2}}
$$

and

$$
x_{v_{L}}^{*}=\rho_{v_{L}}\left(v_{H}\right) \frac{q v_{H} v_{L}^{2}}{\left(v_{H}+v_{L}\right)^{2}}+\left(1-\rho_{v_{L}}\left(v_{H}\right)\right) \frac{q v_{L}}{4} .
$$

Equilibrium beliefs about the opponent's expected effort are 


$$
E_{v_{H}}\left(x_{-i, 1}\right)=\rho_{v_{H}}\left(v_{H}\right) x_{v_{H}}^{*}+\left(1-\rho_{v_{H}}\left(v_{H}\right)\right) x_{v_{L}}^{*}
$$

and

$$
E_{v_{L}}\left(x_{-i, 1}\right)=\rho_{v_{L}}\left(v_{H}\right) x_{v_{H}}^{*}+\left(1-\rho_{v_{L}}\left(v_{H}\right)\right) x_{v_{L}}^{*}
$$

with

$$
E_{v_{H}}\left(x_{-i, 1}\right)>E_{v_{L}}\left(x_{-i, 1}\right) .
$$

In the stage 1 contest, the equilibrium effort of a player of type $\mathbf{h}_{i, 1} \in\left\{v_{H}, v_{L}\right\}$ turns out to be a weighted average of the efforts in the corresponding complete information contests in which the valuations of winning are commonly known, that is, for valuations $\left(v_{i}, v_{-i}\right) \in\left\{\left(v_{i}, v_{H}\right),\left(v_{i}, v_{L}\right)\right\}$. A comparison of $x_{v_{H}}^{*}$ and $x_{v_{L}}^{*}$ in (8) and (9) with $\check{x}_{v_{H}}$ and $\check{x}_{v_{L}}$ as in (5) and (6) shows that subjective probabilities $\rho_{v_{H}}\left(v_{H}\right)$ and $\rho_{v_{L}}\left(v_{H}\right)$ of facing a high-valuation player replace the objective probability $\pi_{\omega}$. Since $\rho_{v_{H}}\left(v_{H}\right)>\rho_{v_{L}}\left(v_{H}\right)$, strong types place more weight on the possibility that $-i$ is a strong type as well, and vice versa. These different weights generate the two different conjectures (10) and (11) about the expected effort of the opponent. This contrasts with the type-independent expectations in Proposition 1.

It is known that it is difficult to solve analytically for the equilibrium of the Tullock contest with incomplete information. Only partial results exist in the literature. ${ }^{15}$ The equilibrium described in Lemma 1 and Proposition 3 considers the case of players who are drawn from the same distribution but can differ in their beliefs about the underlying distribution of types, as a consequence of uncertainty about the true type distribution. A further comparative static result is stated as a corollary. ${ }^{16}$

Corollary 1 The stage 1 equilibrium effort $x_{v_{H}}^{*}$ of strong types is strictly increasing in $\rho_{v_{H}}\left(v_{H}\right)$ and the stage 1 equilibrium effort $x_{v_{L}^{*}}^{*}$ of weak types is strictly decreasing in $\rho_{v_{L}}\left(v_{H}\right)$.

This result is in line with standard intuition in contest theory: players exert more effort if they believe it is likely to meet another player with the same (a similar) valuation. Thus, if strong types believe that it is more likely to be in state $\bar{\omega}$ (with many strong types) they adjust their effort upward. If weak types believe that it is more likely to be in state $\bar{\omega}$ they adjust their effort downward. With (7), both types' efforts in stage 1 go up if the distance $d$ between the two possible states of the world is increased. A higher value of $d$ implies that the true type distribution is more asymmetric; hence, stage 1 beliefs react more strongly to the information about

15 For instance, Malueg and Yates (2004) offer a solution for the equilibrium with ex ante symmetry of players and homogenous beliefs about the distribution from which the opponent is drawn. Serena (2018) offers some comparative static results, but it becomes clear that analytical characterizations of the equilibrium may not be feasible in general.

16 With (8) and (9), Corollary 1 follows from $v_{H}^{2} v_{L}\left(v_{H}+v_{L}\right)^{2}<v_{H} / 4$ and $v_{H} v_{L}^{2} /\left(v_{H}+v_{L}\right)^{2}<v_{L} / 4$. 
the own type and players expect their opponent to be of the same type with higher probability.

Later stages In stages $s \geq 2$, player $i$ 's 'type' is characterized by the own prize valuation and a history of encounters with other players $-i$ with their own valuation $v_{-i}$ and history of previously matched players. If $H_{s}$ contained already $m$ different player types, then any player can be matched with any of these types, such that the set $H_{s+1}$ contains $m^{2}$ elements. In Section B.1 of the Online Appendix we consider properties of stage $s=2$ and establish a ranking of equilibrium efforts (Proposition 6) that demonstrates the potentially countervailing effects of valuation type and experience type on incentives to exert effort. Proposition 6 in the Online Appendix also shows that the stage 2 equilibrium beliefs about the opponent's effort satisfy

$$
E_{\left(v_{H}, x_{v_{H}}^{*}\right)}\left(x_{-i, 2}\right)>E_{\left(v_{H}, x_{v_{L}}^{*}\right)}\left(x_{-i, 2}\right)=E_{\left(v_{L}, x_{v_{H}}^{*}\right)}\left(x_{-i, 2}\right)>E_{\left(v_{L}, x_{v_{L}}^{*}\right)}\left(x_{-i, 2}\right)
$$

where, in the subscript, the first element refers to the player's own valuation and the second element refers to the effort of the stage 1 opponent. Hence, a player's expectation of the opponent's effort is still correlated with the own valuation type so that high-valuation players expect, on average, higher opponent's effort than lowvaluation players.

It is evident that calculating the equilibrium efforts for this problem becomes increasingly intractable in later stages. However, we can consider a limit case where the maximum number of stages grows very large and discuss changes in beliefs and efforts across the stages on an intuitive basis. If the opponents' effort choices remain informative about the type distribution, the impact of the own type on the players' beliefs in stage $s$ becomes less and less important in later stages, as the number of signals obtained increases rapidly (the opponent's effort in stage $s$ is not only informative about this opponent's valuation but also about the opponent's experience in previous stages). In the limit case after a sufficient number of stages, the heterogeneity in beliefs should disappear and all players' beliefs about the share of players with a high prize valuation should converge to the true share $\pi_{\omega}$ (where $\left.\omega \in\{\bar{\omega}, \underline{\omega}\}\right) .{ }^{17}$ Moreover, the players anticipate that their opponent will have the same beliefs with probability (close to) one. As a consequence, the correlation between a player's own effort and the average effort she expects from her stage $s$ opponent identified in (12) and (13) above vanishes in later stages:

\footnotetext{
17 Convergence of beliefs about the true state of the world cannot always be taken for granted. This is discussed in a considerable literature on learning and repeated stochastic fictitious play (see, e.g., Fudenberg and Takahashi 2011). In our context the conditions for learning and belief convergence are quite favorable: effort choices of opponents are informative about these opponents' types (in terms of their types and history), players are randomly drawn from the whole set of players, and there are only two possible states of the world.
} 


$$
\lim _{s \rightarrow \infty} E_{i, s}\left(x_{-i, s} \mid v_{i}=v_{H}\right)=\lim _{s \rightarrow \infty} E_{i, s}\left(x_{-i, s} \mid v_{i}=v_{L}\right) .
$$

Similarly, the average equilibrium effort of high-valuation (low-valuation) players converges to the equilibrium effort of high-valuation (low-valuation) players in a contest in which the players have common beliefs about the type distribution.

To shed light on the expected direction of effort adjustments of strong and weak types, we compare the stage 1 equilibrium efforts $x_{v_{H}}^{*}$ and $x_{v_{L}}^{*}$ given in (8) and (9) to the equilibrium efforts in "very late" stages, where the true share of high types is (basically) common knowledge as in Proposition 1 . The latter are denoted by $\breve{x}_{v_{H}}$ and $\breve{x}_{v_{L}}$ and we assume an interior equilibrium $\left(\breve{x}_{v_{H}}, \breve{x}_{v_{L}}\right) \in(\underline{x}, \bar{x})^{2}$.

Proposition 4 (i) If the state of the world is $\omega=\bar{\omega}$ (with many strong types), then $\breve{x}_{v_{L}}<x_{v_{L}}^{*}$. (ii) If the state of the world is $\omega=\underline{\omega}$ (with many weak types), then $\breve{x}_{v_{L}}>x_{v_{L}}^{*}$ and $\breve{x}_{v_{H}}<x_{v_{H}}^{*}$.

Proposition 4 provides a further theoretical foundation for the empirical analysis below. In particular, it makes a prediction on the adjustments of efforts of low and high types in late stages as compared to stage 1 conditional on the shape of the type distribution. More informally, if the true distribution of types is such that there are many low-valuation players, the players' beliefs about the share of strong types are corrected downwards in later stages as compared to the players' beliefs at stage 1 . This holds in particular for high-valuation players who initially believe that there are more strong types (compare (7)). As a consequence of this updating, the average effort of strong types should be decreasing and the average effort of weak types should be increasing in later stages. Conversely, if the distribution of types is such that there are many high-valuation players, the average effort of strong types should be increasing and the average effort of weak types should be decreasing in later stages. ${ }^{18}$ These different dynamics reflect the intuition that players increase their effort if they learn that it is likely to face an opponent with a similar valuation, and reduce their effort if they learn that the contest is likely to be asymmetric.

An exit option and self-selection In order to identify selection effects in the data, a modified version of the game allows players to exit the game at the end of stage 1 in case the conflict has not yet been resolved. Formally, after observing the outcome of the stage 1 contest, all players simultaneously and independently decide whether to exit the game. In case of exit a player receives a fixed payment $b$ but does no longer participate in stages $2, \ldots, n$. For the players who do not exit the game continues with possible contest stages $s=2, \ldots, n$ within the population of players who did not exit. If all but a set of players with mass zero exit, the game ends for all players.

\footnotetext{
18 In theory the ranking between effort $\breve{x}_{v_{H}}\left(\pi_{\bar{\omega}}\right)$ and stage 1 effort $x_{v_{H}}^{*}$ is ambiguous since the strong reduction in $\breve{x}_{v_{L}}\left(\pi_{\bar{\omega}}\right)$ as compared to $x_{v_{L}}^{*}$ weakens the high-valuation types' incentive to exert effort. In state $\bar{\omega}, \breve{x}_{v_{H}} \leq x_{v_{H}}^{*}$ occurs, however, only for "extreme" parameter values; for details see the proof of Proposition 4.
} 
Proposition 5 Suppose that exit is possible at the end of stage 1. For an exit payment $b \in\left[b_{L}, b_{H}\right]$, there is a perfect Bayesian equilibrium in which all players with valuation $v_{L}$ exit and all players with valuation $v_{H}$ do not exit, where $b_{L}$ and $b_{H}$ are given by

$$
b_{L} \equiv\left[q \frac{\max \left\{\tilde{x}_{L}, \underline{x}\right\}}{\max \left\{\tilde{x}_{L}, \underline{x}\right\}+\frac{q v_{H}}{4}} v_{L}-\max \left\{\tilde{x}_{L}, \underline{x}\right\}\right] \sum_{t=2}^{n}(1-q)^{t-2}
$$

with $\tilde{x}_{L} \equiv q\left(-v_{H}+2 \sqrt{v_{L} v_{H}}\right) / 4$ and

$$
b_{H} \equiv \frac{q v_{H}}{4} \sum_{t=2}^{n}(1-q)^{t-2}>b_{L} .
$$

At stages $s=2, \ldots, n$ (if reached), all players believe that their opponent has a valuation $v_{H}$ with probability one and equilibrium efforts are equal to

$$
x_{s}^{*}=\frac{q v_{H}}{4} .
$$

For intermediate values of the exit option, there is an equilibrium in which all weak types exit so that the population of players in stages $s \geq 2$ consists of strong types only. The value $b_{H}$ represents the expected continuation payoff of strong types; the constraint $b \geq b_{L}$ ensures that weak types do not want to deviate from this equilibrium. $^{19}$

In the equilibrium in which weak types exit and strong types remain, average effort in stages $s \geq 2$ is strictly higher than average stage 1 effort, due to two effects. ${ }^{20}$ First, there is the direct self-selection effect that causes the population in stages $s \geq 2$ to be composed only of players who care strongly about winning. Second, since in stages $s \geq 2$ strong types correctly anticipate that their opponent will be a strong type, they further increase their effort as compared to stage 1 .

\footnotetext{
19 This separating equilibrium with selection described in Proposition 5 need not be unique. There is always a trivial equilibrium in which all players exit because they expect that all other players exit. And there can be equilibria with pooling, depending on the size of the exit payment. For instance, an exit payment $b$ that is very close to $b_{L}$ is also compatible with an equilibrium in which no player exits, due to the complementarity of exit decisions. If many weak types remain active, this makes it more likely that other players are matched with a weak type, which makes it more attractive for other weak players to remain active.

20 By Corollary 1, the stage 1 effort of a high type is strictly lower than $q v_{H} / 4$ and approaches $q v_{H} / 4$ if the probability $\rho_{v_{H}}\left(v_{H}\right)$ that a strong type assigns to meeting another strong type approaches one. Similarly, stage 2 effort $x_{\left(v_{H}^{*}, x_{v_{H}}^{*}\right)}$ is strictly lower than $q v_{H} / 4$ so that $x_{s}^{*}$ in (17) is also strictly larger than all types' equilibrium stage 2 effort in the framework without exit option. By an equivalent argument, $x_{s}^{*}$ in (17) is strictly larger than the equilibrium efforts $\breve{x}_{v_{H}}\left(\pi_{\bar{\omega}}\right)$ and $\breve{x}_{v_{H}}\left(\pi_{\underline{\omega}}\right)$ in case all players know the true share of strong and weak types.
} 


\subsection{Summary of the main predictions}

The theoretical analysis provides the basis of four main testable predictions. First, ignoring potential type heterogeneity and population uncertainty, efforts should be constant across all stages and beliefs should be type-independent (Proposition 1). Second, if there is unobservable heterogeneity in the (intrinsic) motivation to win and players are ex ante uncertain about the distribution of these player types in the population, then the individual beliefs about the opponent's effort are positively correlated with the own effort in early stages of the game. The correlation should become weaker in later stages of the game (compare Lemma 1 and Proposition 6 in Section B.1 of the Online Appendix as well as the discussion around (14)). Third, if the true type distribution consists of more weak types than expected (with a low intrinsic motivation), weak types' effort should go up and strong types' effort should go down in later stages, as compared to stage 1 (Proposition 4). The opposite dynamics should prevail if the population consists of more strong types than expected. Forth, if exit is possible at the end of stage 1, weak types should exit and strong types should remain so that average effort in stages $2, \ldots, n$ is strictly higher than average stage 1 effort (Proposition 5).

The intuition for the dynamics of efforts closely follows a standard contest logic which is due to the non-monotonicity of best-reply functions. Weak types should be discouraged when learning that there are many strong opponents, and encouraged when learning that there are many weak opponents. Strong types should become more competitive when learning that there are many strong opponents, and should be "appeased" when learning there are many weak opponents. Together with the direction in which the beliefs are adjusted in the respective population, this explains the basic mechanism behind Proposition 4 .

\section{Experimental design}

To emphasize the importance of accounting for unobserved type heterogeneity, the experimental treatments use the common approach of symmetric monetary incentives (a given contest prize) and common knowledge about these. As explained above, however, we expect significant preference heterogeneity even under symmetric monetary incentives. Thus, our experimental strategy picks up on naturally occurring heterogeneities (as present in most experiments) in order to contrast the structurally different theory predictions with and without incomplete information and population uncertainty.

\subsection{Treatments}

The baseline experimental treatment BASE corresponds to the theory framework outlined above and investigates the importance of accounting for unobserved heterogeneity in preference types and belief formation, as opposed to the benchmark prediction based on complete information. In the experiment, the individuals 
compete in up to $n=5$ stages about a prize of monetary value $v=450$ by choosing investments $x_{i, s}$ from the set $\{1,2, \ldots, 450\}$ at each stage $s$ that is reached. Together with the choice of the own effort, each individual has to state the effort she expects from her opponent at this stage (as a number between 1 and 450); the stated beliefs $E_{i, s}\left(x_{-i, s}\right)$ are not displayed to other players. After the effort choices have been made at stage $s$, the individuals observe the investment $x_{-i, s}$ of their opponent and a "lottery wheel" determines whether one of the two players is allocated the prize or whether the game proceeds to the next stage; this outcome is observed, too. ${ }^{21}$ The exogenous probability that the prize is allocated in a given stage is $q=1 / 3$, which is supposed to balance a reasonable chance of winning the prize with a sufficiently high probability of continuation and, hence, possible dynamics. At each stage, the individuals are randomly re-matched in pairs. Once the game ends because the prize has been allocated or stage $s=5$ has been completed without prize allocation, each individual is displayed her own payoff.

This design with random matching as well as anonymity and non-identifiability of participants stays close to the theory as long as the subjects in the laboratory do not believe that their actions have informational content that feeds back into their own future encounters. The probability that, in a given session, a player interacted with the same player more than once is not zero in our setup, but the respective player would not know if/when meeting a particular opponent again, which should make quasi-repeated play effects rather unlikely.

Whereas dynamic conflict games typically involve explicit or implicit participation decisions, the BASE treatment removes such considerations by design. As the main experimental variation, the EXIT treatment therefore adds the possibility of exit and, hence, an explicit continuation decision. Based on this experimental variation, we can identify possible effort escalation caused by self-selection as a consequence of unobserved preference heterogeneity. As in the modified theory framework described above, the individuals have the option to exit the game at the end of stage 1, after observing the stage 1 efforts and outcome and in case the prize has not yet been allocated at stage $1 .{ }^{22}$ Individuals make this choice between "exit" and "remain" simultaneously and independently. Denote the stage 1 pair of players by $(i,-i)$. If both $i$ and $-i$ choose to exit then the game ends for both individuals with an exit payment of 60 points each (minus the individual cost of stage 1 effort). If both individuals $i$ and $-i$ choose to remain then both enter into stage 2 (where new pairs of subjects are randomly formed). If one individual chooses to exit and her stage 1 opponent chooses to remain then a coin flip decides on whether both subjects exit

\footnotetext{
${ }^{21}$ The lottery wheel is a circle area with colored segments that represent the two players' win probabilities $q p_{i, s}$ and $q p_{-i, s}$ as well as a gray segment that corresponds the probability $1-q$ that the prize is not allocated at stage $s$. An arrow that rotates around the circle area determines the outcome of stage $s$.

${ }^{22}$ The one-time nature of the exit option facilitates the identification of a treatment effect by allowing for a binary, before-after comparison (similar to an entry decision). It only requires to fix one value for the outside option instead of determining appropriate stage-dependent outside options. In addition to a standard entry choice, our design allows to analyze whether the experience in stage 1 affects the subsequent choice of exit.
} 
or enter into stage $2 .^{23}$ Apart from adding this exit option, the sequence of actions in the EXIT treatment is exactly as in the BASE treatment. The payment in case of exit is chosen to be lower than the equilibrium expected continuation payoff in the benchmark case with symmetric players who maximize their monetary payoffs so that in the latter case no player should exit in equilibrium.

Our choice of symmetric monetary incentives allows to attribute any heterogeneity in behavior to differences in unobserved (preference) characteristics. This comes at the cost of not being able to identify different types of players based on an observable objective function but having to rely on individual choices in order to distinguish different behavioral types of players. This latter approach to classifying types, which is common when one expects factors beyond monetary payoffs to matter, would be appropriate even when imposing heterogeneity in extrinsic (incentivized) motivations as another layer of heterogeneity: even with imposed differences in effort costs, for instance, the sets of individuals with identical monetary incentives would have to be expected to differ along important preference characteristics. The vast majority of contest experiments has shown that behavior cannot be understood without incorporating motives beyond monetary payoff maximization. ${ }^{24}$

\subsection{Experimental procedures}

The experiment was conducted at econlab Munich in two waves and 19 sessions in total (with typically 24 subjects per session). A first wave in April 2016 involved 4 sessions of each treatment BASE and EXIT; in this wave, the elicitation of beliefs was not incentivized to reduce complexity. A second wave in May 2019 involved 4 sessions of the BASE treatment for which the elicitation of beliefs was incentivized, plus 4 BASE sessions with nonincentivized beliefs and 3 EXIT sessions to ensure comparability of the two waves. The subjects (422 in total) were typically students of Munich universities. ${ }^{25}$ Each subject took part in exactly one session. In each treatment, the respective multi-stage contest was played for 15 times. In other words, each subject played the same game (with up to 5 stages $s$ ) in 15 rounds $r$.

At the beginning of each session each subject was shown a video on the computer screen in which the experimental instructions (also distributed as hard copy) were

\footnotetext{
23 This procedure guarantees that there is an even number of subjects in all stages, and is easy to explain to the subjects. It has the advantage that it yields additional experimental variation by extending the sample in later stages to subjects who actually revealed a preference for exit.

${ }^{24}$ We also implemented an experimental variation where the individuals repeatedly face the same opponent and can, hence, learn about a particular opponent's type. The experimental results for this FIXED treatment are reported in Section B.8 of the Online Appendix. From a theory point of view, updating about the specific opponent's type adds a strategic link between the stages. Due to the resulting signaling problems, the equilibrium is known to be difficult to solve for even under restrictive assumptions. Münster (2009) identifies strategic "sandbagging" in a model with two stages and common beliefs about the type distribution where $v_{i} \in\left\{0, v_{H}\right\}$.

${ }^{25}$ The subjects were recruited using the software ORSEE (Greiner 2004). About one half of the students were female, $20 \%$ studied economics or a related field, and the average age was 23 . The experiment was programmed using z-Tree (Fischbacher 2007). For the experimental instructions see Section C of the Online Appendix.
} 
read aloud. Then, the subjects had to answer a few control questions to ensure they understood the rules of the experiment. After the 15 rounds of the main experiment, we conducted an extended post-experimental questionnaire which, apart from socioeconomic information and questions about the experiment, elicited measures for risk preferences, distributional preferences, ambiguity aversion, loss aversion, and cognitive reflection. At the end of the experiment, 3 out of the 15 rounds were randomly selected for payment and a subject's total points won minus her investments $x_{i, s}$ were summed up. In the sessions in which the stated beliefs were incentivized, one further round was selected (different from the 3 rounds for which the contest outcome was paid) and a subject obtained 450 points if her stated beliefs in this additional round deviated, on average, by 5 points or less from the actual opponent effort (that is, if on average $\left|E_{\text {irs }}\left(x_{-i r s}\right)-x_{-i r s}\right| \leq 5$ in this round). Moreover, one of the incentivized post-experimental tasks were randomly selected for payment. The resulting amount of money was converted to Euros at the rate $50: 1$ and added to (or subtracted from) an endowment of 10 Euros. On average a session lasted 90 minutes and the average payment was 17.70 Euros plus a show-up fee of 6 Euros.

For the experimental sessions, the randomization of whether or not in a given stage $s \in\{1, \ldots, 5\}$ of round $r \in\{1, \ldots, 15\}$ the prize would be allocated (with the exogenous probability $q=1 / 3$ ) was conducted (but not announced) before the start of the first session and was kept the same across all treatments, sessions, and subject pairs. ${ }^{26}$ In other words, the number of stages to be played within round $r \in\{1, \ldots, 15\}$ was the same for all subjects. This ensures that learning about the game and the numbers of signals obtained about the distribution of types are identical across treatments and sessions at any stage $s$ of round $r$. The random re-matching took place in subgroups (typically 8 subjects, although the precise size of the matching groups was not made explicit) in order to gain more independence of observations and allow us to investigate learning dynamics across different populations. ${ }^{27}$

\section{Results}

\subsection{Overview of the main results}

Do the individuals adjust their effort in later stages of the game, and if yes, is there a tendency to escalate or to de-escalate for different player types? The left panel of Fig. 1 provides a first answer to this question by plotting average efforts in the five stages. The graph shows that in the BASE treatment there is a slight upward trend

\footnotetext{
${ }^{26}$ Before running the experiment we independently drew $15 \times 5$ random numbers $y_{r s}$ from a uniform distribution on the unit interval so that (i) a number $y_{r s} \leq 1 / 3$ indicated that in stage $s$ of round $r$ (if reached) the prize would be allocated based on the player's effort choices and (ii) a number $y_{r s}>1 / 3$ indicated that in stage $s$ of round $r$ (if reached) the prize would not be allocated but the game would proceed to stage $s+1$ (or end if $s=5$ ).

27 The structure of the randomization of the continuation probabilities made sure that the size of the matching groups remained constant across all stages; the players within a matching group ended a round all at the same stage (in the treatments without exit option).
} 
in average efforts. In the EXIT treatment, stage 1 efforts are comparable, but from stage 2 onward (after exit was possible) there is an upward jump in average efforts (in line with Proposition 5). The higher variance in efforts in the EXIT treatment may be caused by the lower number of observations in stages 2-5 (due to exit of a substantial share of individuals).

A regression analysis confirms the finding suggested by the left panel of Fig. 1: we can reject the complete-information prediction of Proposition 1 (the prediction ignoring population uncertainty) that average efforts do not change across the treatments, even when disregarding possibly different dynamics of different player types. Table 3 in Section B.3 of the Online Appendix summarizes the corresponding results from a set of random-effects regressions based on individual efforts, which estimate the average change in efforts across the stages. ${ }^{28}$

Result 1 Average efforts significantly change across the stages, in contrast to the complete-information benchmark. The effect is strongest in the EXIT treatment where average efforts go up by $23 \%$ after exit was possible.

The right panel of Fig. 1 reveals a considerable heterogeneity in individual behavior by plotting the distribution of the individuals' average effort choice in early rounds (rounds 1-5). Accounting for this evident, nonincentivized heterogeneity, which is a common finding in contest experiments, we turn to the main analysis of type-dependent effort adjustments. Since the data from the two different waves of the experiment yields identical conclusions, the subsequent analysis pools the sessions from the two waves.

\subsection{On individual efforts}

Whereas the theory above makes no definite statement about average adjustments of efforts, it predicts differential effects for different 'types' of players with different intrinsic motivations: players classified as 'weak' and players classified as 'strong'. To allow for such (unobserved) heterogeneity we use an individual's effort choice as a proxy for her valuation of winning. We separate the individuals into strong and weak types according to whether their average effort in rounds 1-5 (as plotted in the right panel of Fig. 1) is below or above the treatment average in those rounds. ${ }^{29}$

\footnotetext{
${ }^{28}$ Like the main estimations of type-dependent effort adjustments below, the regressions in Table 3 use the effort $x_{i r s}$ of individual $i$ in stage $s$ of round $r$ as the dependent variable and test whether there is a linear trend in efforts (measured by the variable "Stage ${ }_{s-1}$ ") and a change in efforts from stage 2 on (measured by the indicator variable $\mathbf{1}_{s \geq 2}$ ). In BASE there is a small and weakly significant upward trend in average efforts of about 0.78 points per stage (see estimation 1). In EXIT, efforts increase in stage 2 by 3.7-5 points as compared to average stage 1 effort (see estimations 2 and 3); the estimated coefficient of the interaction term "EXIT $\times \mathbf{1}_{s \geq 2}$ " in estimation 4 shows an increase in average efforts of 8.15 points in stage 2, relative to the baseline. Also, there is no further trend in average efforts in EXIT from stage 2 onwards (see the sum of the coefficients of "Stage ${ }_{s-1}$ " and its interaction with "EXIT" in estimation 4; $p$ value is 0.833$)$.

29 In BASE, the average share of 'strong types' per matching group (population) is 0.418 , with a minimum of 0.125 , median of 0.375 , maximum of 0.875 and a standard deviation of 0.235 (36 matching
} 

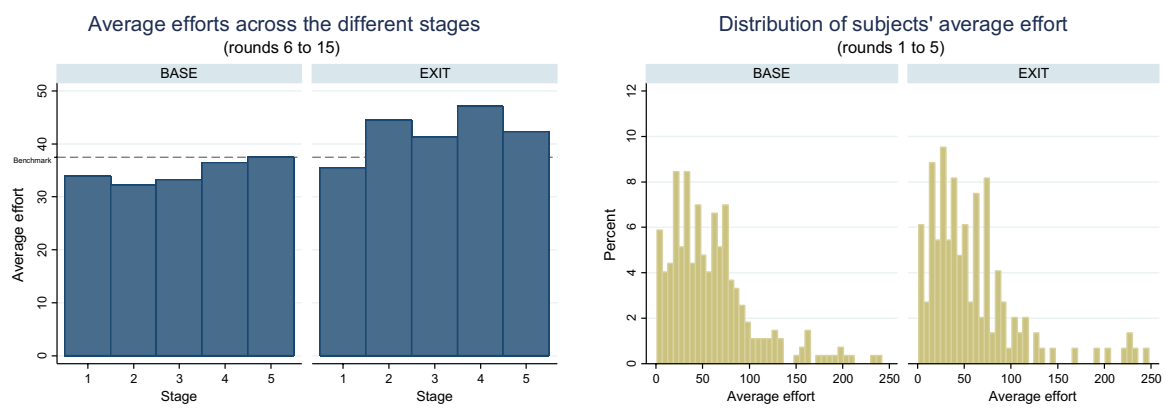

Fig. 1 Left panel: average effort choices in stages $1-5$ by treatment; right panel: distribution of 'types' by treatment

With this classification as weak or strong type, we estimate efforts $x_{i r s}$ (of individual $i$ in stage $s$ of round $r$ ) across the stages based on the data of rounds 6-15, interacting the main explanatory variables "Stage ${ }_{s-1}$ " and $\mathbf{1}_{s \geq 2}$, respectively, with the proxy for a player's type (an indicator variable $\mathbf{1}_{\text {strong type }}$ ). The variable "Stage ${ }_{s-1}$ " is equal to $s-1$ so that the coefficient of "Stage $e_{s-1}$ " measures the average per-stage change in effort and the intercept estimates average effort in stage 1 . The indicator variable $\mathbf{1}_{s \geq 2}$ for the observations from stages $s \geq 2$ identifies an effect of the exit option in the EXIT treatment.

The estimation results are presented in Table 1 . To simplify the exposition we present separate estimations for the treatments and focus on a linear trend in efforts (variable "Stage ${ }_{s-1}$ ") in BASE and a discontinuity in efforts in stage 2 (indicator variable $\left.\mathbf{1}_{s \geq 2}\right)$ in EXIT. All estimations control for the stated beliefs $E_{\text {irs }}\left(x_{-i r s}\right)$ and $\left(E_{i r s}\left(x_{-i r s}\right)\right)^{2}$ to capture the predicted non-monotonicity of the best reply functions. Moreover, we include dummy variables for the rounds $r$, the different sessions, and individual-specific control variables obtained from the post-experimental questionnaire.

Estimation (1) on the BASE treatment focuses on behavior from rounds 6-15 where subjects have gained some experience with the multi-stage setup. The large and significant coefficient of the indicator variable $\mathbf{1}_{\text {strong type }}$ shows that those subjects classified as strong types by their effort in early rounds also choose higher effort in later rounds, compared to weak types. The positive and significant coefficient of the variable "Stage ${ }_{s-1}$ " measures an increase of efforts across stages by weak types. For those types, the estimated average escalation of efforts per stage is 1.53 points. Moreover, the adjustment of efforts is significantly different for strong and weak types, as indicated by the coefficient of the interaction term $\mathbf{1}_{\text {strong type }} \times$ Stage $_{s-1}$. For the strong types, however, efforts do not change across the stages: the sum of "Stage ${ }_{s-1}$ " and its interaction with the indicator variable $\mathbf{1}_{\text {strong type }}$ is close to zero

Footnote 29 (continued)

groups in total). In EXIT, the average share of 'strong types' per matching group (population) is 0.353 , with a minimum of 0.125 , median of 0.333 , maximum of 0.875 and a standard deviation of 0.218 (21 matching groups in total). 
Table 1 Individual effort over stages 1-5: strong versus weak types

\begin{tabular}{|c|c|c|c|c|}
\hline \multirow[t]{2}{*}{ Indep. var. } & (1) & (2) & (3) & (4) \\
\hline & BASE & $\begin{array}{l}\text { BASE (early } \\
\text { rounds) }\end{array}$ & BASE (late rounds) & EXIT \\
\hline Constant & $31.042 * * *(3.083)$ & $25.788 * * *(3.909)$ & $32.446 * * *(3.446)$ & $16.748(11.066)$ \\
\hline $\mathbf{1}_{\text {strong type }}$ & $22.322 * * *(3.440)$ & $33.188 * * *(4.408)$ & $19.021 * * *(3.612)$ & $26.630 * * *(4.728)$ \\
\hline Stage $_{s-1}$ & $1.533 * * *(0.463)$ & $1.920 * * *(0.533)$ & $1.466 * * *(0.529)$ & \\
\hline $\mathbf{1}_{\text {strong type }} \times$ Stage $_{s-1}$ & $-1.825 * *(0.914)$ & $-4.112 * * *(1.440)$ & $-1.473^{*}(0.821)$ & \\
\hline $\mathbf{1}_{s \geq 2}$ & & & & $4.765^{* *}(2.015)$ \\
\hline $\mathbf{1}_{\text {strong type }} \times \mathbf{1}_{s \geq 2}$ & & & & $-2.815(4.543)$ \\
\hline Beliefs $E_{i r s}\left(x_{-i r s}\right)$ & $0.260 * * *(0.043)$ & $0.298 * * *(0.044)$ & $0.216^{* * *}(0.037)$ & $0.310 * * *(0.050)$ \\
\hline $\operatorname{Beliefs}\left(E_{i r s}\left(x_{-i r s}\right)\right)^{2}$ & $-0.001 * * *(0.000)$ & $-0.001 * * *(0.000)$ & $-0.001 * * *(0.000)$ & $-0.001(0.000)$ \\
\hline Round fixed effects & Yes & Yes & Yes & Yes \\
\hline $\begin{array}{l}\text { Session fixed } \\
\text { effects }\end{array}$ & Yes & Yes & Yes & Yes \\
\hline Socioeconomics & Yes & Yes & Yes & Yes \\
\hline Observations & 7946 & 4932 & 4658 & 2878 \\
\hline
\end{tabular}

Random-effects regressions; SE in parentheses, clustered at the level of matching groups; *** $(* *, *)$ significant at $1 \%(5 \%, 10 \%)$. Estimations (1) and (4): data from rounds 6-15; estimation (2): data from early rounds (rounds $3-9$ ); estimation (3): data from late rounds (rounds $10-15$ ). $\mathbf{1}_{\text {strong type }}=1$ if subject $i$ 's average effort in rounds 1-5 higher than average effort of all subjects in rounds $1-5$ of the respective treatment, and $\mathbf{1}_{\text {strong type }}=0$ otherwise. "Stage o $_{s-1}$ " is equal to stage number $s-1 . \mathbf{1}_{s \geq 2}=1$ if stage $\geq 2$, and $\mathbf{1}_{s \geq 2}=0$ otherwise

and insignificant ( $p$ value is 0.728 ). Hence, the observed increase in efforts in the treatment without exit option is driven by the weak types. ${ }^{30}$

Estimations (2) and (3) show that the described effort dynamics in the BASE treatment are stronger in earlier rounds where beliefs are supposed to adjust more strongly, and weaken in later rounds where adjustments of beliefs should diminish. The relevant coefficients of "Stage ${ }_{s-1}$ " and $\mathbf{1}_{\text {strong type }} \times$ Stage $_{s-1}$ change in terms of size and significance in early as compared to late rounds (independent of the exact set of rounds classified as "early" or "late" rounds). In early rounds (estimation 2), the observed downward adjustment of strong types' effort is borderline significant ( $p$ value is 0.100 ).

\footnotetext{
${ }^{30}$ The main findings are robust to excluding the individual control variables, the round or the session dummies or including dummy variables for the matching groups. They are also robust to extending the sample of observations to earlier rounds. Only round 1 turns out to be structurally different, exhibiting a strong downward adjustment of efforts which, in the first contests played, are more than twice the average of later rounds. Finally, the main results are very similar when using average efforts in late rounds (under experienced behavior) in order to classify types as 'strong' or 'weak,' using the median effort of a treatment as the threshold for the type classification or using a continuous variable for player types.
} 
Result 2 In the BASE treatment, the increase in efforts in later stages is caused by weak types. For strong types we find no such increase in efforts.

How do the results on efforts presented so far relate to the theory of updating of beliefs under uncertainty about the type distribution? According to the conjecture based on Proposition 4, the adjustments of efforts depend on underlying type distribution: the weak types' average effort should be increasing and the strong types' average effort should be decreasing across stages if the true state of the world is state $\underline{\omega}$ with many low-valuation types. Using a subject's average effort in rounds 1-5 as a proxy for the subject's valuation type, the right panel of Fig. 1 has shown that the empirical type distribution leans toward weak types (about $60 \%$ of the subjects are classified as 'weak'), suggesting that the type-dependent adjustments of efforts observed across stages are in line with what the theory predicts for the underlying type distribution with many weak types (compare Proposition 4(ii)). ${ }^{31}$

Also, it speaks in favor of the theory that the adjustment effects are driven by earlier rounds where the informational value of observing the opponent's effort in a given stage is larger. ${ }^{32}$ Similarly, if we investigate effort dynamics across contest subgames played (across the rounds of the experiment), we find increasing efforts of weak types and decreasing efforts of strong types for the subsample of earlier rounds and no significant adjustments for later rounds. Nevertheless, the dynamics appear quite persistent, which would suggest that updating of beliefs may occur across stages not only in the first rounds, possibly in the same way in which too much weight is placed on the own type (we will come back to the subjects' stated beliefs in Sect. 4.4). Similarly, a "restart effect" in new rounds could favor adjustments of beliefs and efforts even within later rounds.

There are, however, two caveats to be made: First, our type classification relies on behavior in early interactions, rather than being based on pre-experimental tasks.

\footnotetext{
31 Since the subjects interacted within subsets of participants (matching groups) only, we can also employ heterogeneity in the type distributions across different populations and re-run the estimations of Table 1 by including a three-way interaction term of $\mathbf{1}_{\text {strong type "Stage }}$ " " and a variable "ShareStrong ${ }_{g}$ " measuring the share of players classified as 'strong' within the respective matching group. The results are summarized in Table 6 in Section B.7 of the Online Appendix, which shows that the weak types' increase of efforts across stages is most pronounced in matching groups with many weak types but does not occur in matching groups with many strong types (compare estimations 1 and 2 as well as the coefficients of "Stage ${ }_{s-1}$ " and its interaction with "ShareStrong ${ }_{g}$ " in estimation 3). For strong types, the decrease of efforts across stages is most pronounced in matching groups with many weak types; efforts tend to increase in matching groups with many strong types when employing interaction terms (compare, for instance, the sum of the coefficients of Stage ${ }_{s-1} \times$ ShareStrong $_{g}$ and its interaction with $\mathbf{1}_{\text {strong type }}$ in estimation 3). Although the coefficients on the interaction terms with the variable for the share of strong types in estimations 3 and 4 are imprecisely measured, these different dynamics are qualitatively in line with the predictions of Proposition 4, providing further support for the importance of updating of beliefs about the underlying type distribution.

32 One explanation for why the adjustments predominantly emerge for weak types goes back to the intuition behind the theory considerations. For weak types, the incentive to exert effort increases due to the direct effect of learning that there are many weak types and due to the indirect effect of a reduction of strong types' efforts. For strong types, the direct effect (learning that there are many weak types) reduces the incentive to exert effort, but the indirect effect of an increase in weak types' effort works in the opposite direction and increases the incentive to exert effort.
} 
Table 2 Individual choice whether to exit

\begin{tabular}{lllll}
\hline Dependent variable: & choice of exit $\left(\right.$ exit $_{i r 1}=1$ if exit) & & \\
\hline Indep. var. & $(1)$ & $(2)$ & $(3)$ & $(4)$ \\
& EXIT & EXIT & EXIT & EXIT \\
\hline Constant & $0.406(1.297)$ & $0.146(1.350)$ & $0.470(1.298)$ & $0.083(0.596)$ \\
$\mathbf{1}_{\text {strong type }}$ & $-1.497^{* * *}(0.482)$ & $-1.542^{* * *}(0.486)$ & $-1.548^{* * * *}(0.477)$ & $-1.748^{* * *}(0.501)$ \\
$-i^{\prime}$ ' effort $x_{-i r 1}$ & $-0.002(0.004)$ & & & \\
$\mathbf{1}_{x_{-i r 1}>E_{i r 1}\left(x_{-i r 1}\right)}$ & & $0.718^{* * *}(0.201)$ & & $0.654^{* * * *}(0.213)$ \\
$\left(x_{-i r 1}-E_{i r 1}\left(x_{-i r 1}\right)\right) / x_{-i r 1}$ & & $0.023^{* *}(0.011)$ & \\
$\mathbf{1}_{\text {late }}$ & & & & $0.332(0.368)$ \\
$\mathbf{1}_{\text {strong type }} \times \mathbf{1}_{\text {late }}$ & & & & $0.279(0.287)$ \\
$\mathbf{1}_{x_{-i r 1}>E_{i r 1}\left(x_{-i r 1}\right)} \times \mathbf{1}_{\text {late }}$ & Yes & Yes & Yes & $-0.293(0.294)$ \\
Round fixed effects & Yes & Yes \\
Session fixed effects & Yes & Yes & Yes & Yes \\
Socioeconomics & Yes & Yes & 1184 & Yes \\
Observations & 1184 & 1184 & 1776 \\
\hline
\end{tabular}

Random-effects logistic regressions; SE in parentheses, clustered at the level of matching groups; *** $(* *, *)$ significant at $1 \%(5 \%, 10 \%)$. Estimations (1)-(3): data from rounds 6-15; estimation (4): data from rounds $1-15 . \mathbf{1}_{\text {strong type }}=1$ if subject $i$ 's average effort in rounds $1-5$ higher than average effort of all subjects in rounds $1-5$ of the respective treatment, and $\mathbf{1}_{\text {strong type }}=0$ otherwise. $\mathbf{1}_{\left.x_{-i \mathrm{r} 1}\right) E_{i \mathrm{ir} 1}\left(x_{-i \mathrm{r} 1}\right)}=1$ if opponent's actual stage 1 effort $>$ stated beliefs about opponent's effort, and $\mathbf{1}_{x_{-i r 1}>E_{i r 1}\left(x_{-i r 1}\right)}=0$ otherwise. $\mathbf{1}_{\text {late }}=1$ if round $r=8, \ldots, 15$ and $\mathbf{1}_{\text {late }}=0$ otherwise

This has the disadvantage that we do not have a direct measure for, say, a 'joy of winning'. Nevertheless, we see this approach based on choices as more effective since intrinsic motivations are expected to be specific to the game played and preference heterogeneity arises along multiple dimensions so that a single preference measure may induce misleading interpretations. Second, in addition to learning about the population of opponents, the individuals may learn about the game (about their own 'preference type'). A separation of these two types of learning poses an empirical challenge but we are confident that by dropping the first contest interactions we reduce the role of the latter. Altogether, we do not want to claim that the theory of updating of beliefs is the sole mechanism that drives the observed results of escalation. Beyond this theory that focuses on type heterogeneity, there may be a general, type-independent tendency to escalate efforts in later stages. ${ }^{33}$

\footnotetext{
${ }^{33}$ Such a complementary explanation of escalation can be based on subjective probability weighting in the spirit of Quiggin (1982), Yaari (1987), Prelec (1998) (see Section B.2 of the Online Appendix) An example calculated there for parameter values $v=450$ and $q=1 / 3$ in a given stage and subjective probability weights as suggested in Baharad and Nitzan (2008) yields equilibrium effort choices $\check{x}_{i, 1}=18.33$, $\breve{x}_{i, 2}=18.35, \breve{x}_{i, 3}=18.68, \check{x}_{i, 4}=19.98$, and $\check{x}_{i, 5}=25.25$. Probability weighting cannot straightforwardly explain differential effects on escalation for subjects with strong and weak intrinsic motivation, however.
} 


\subsection{On self-selection}

The estimations on effort choices demonstrated the strongest adjustment effect in the EXIT treatment. To understand the role of selection we run logistic randomeffects regressions on individual $i$ 's choice exit ${ }_{i r 1}$ whether to exit the game at the end of stage 1 of round $r .{ }^{34}$ The estimation results presented in Table 2 confirm a self-selection effect based on the propensity to invest much effort: the probability to exit is significantly lower for strong types (the predicted marginal effect of $1_{\text {strong type }}$ is in the range between -0.15 and -0.24 , depending on the exact specification). This biases the sample in stages $s \geq 2$ toward strong types so that equilibrium effort should go up as compared to stage 1 (compare Proposition 5), providing an explanation for Result 1.

The estimation results in Table 2 also show that the effort of the stage 1 opponent has no significant effect on the probability to exit (compare estimation 1), which is plausible given the random re-matching of player pairs in the subsequent stages. The difference between the stated beliefs $E_{i r 1}\left(x_{-i r 1}\right)$ about the opponent's effort and the actually observed effort $x_{-i r 1}$, however, can explain the choice to exit: those players who underestimated the opponent's effort are more likely to exit. This holds when using an indicator variable for whether actual opponent's effort $x_{-i r 1}$ is higher than stated beliefs $E_{i r 1}\left(x_{-i r 1}\right)$ (estimation 2 of Table 2 ; $p$ value $<0.000$ ) or when including the (relative) difference of actual effort $x_{-i r 1}$ and stated beliefs (estimation 3 of Table 2; $p$ value is 0.028 ). Even when players are randomly re-matched in later stages, the update in beliefs following the "negative surprise" of unexpectedly high opponent's effort can make individuals revise their expectations about payoffs to be obtained in later stages and, hence, affect their choice of exit. ${ }^{35}$

Result 3 In the EXIT treatment, weak types are more likely to exit. Moreover, individuals who are negatively surprised by high opponent's effort in stage 1 are more likely to exit.

Estimation (4) in Table 2 investigates differences in early as compared to late rounds. The self-selection effects measured by the indicator variable for strong types become slightly weaker in later rounds where the subjects have gained some experience but is significant at the $1 \%$-level both in early and in late rounds. The effects of beliefs captured by underestimating the opponent's effort become slightly weaker and less significant in later rounds where updating of beliefs is supposed to be less

\footnotetext{
34 The probability of a choice of "exit" is equal to 0.5 in rounds $1-5$ and equal to 0.48 in rounds 6-15.

35 The results in Table 2 are robust to excluding the individual control variables, the round or the session dummies, including dummies for the matching groups or using alternative definitions for 'strong types' such as a classification based on median efforts, on efforts in late rounds or a continuous variable (average effort in early rounds or in stage 1 of the respective round). Among the individual-specific control variables, the variables capturing risk aversion, loss aversion and impulsiveness significantly positively affect the likelihood to exit.
} 
important. ${ }^{36}$ To summarize, the increase in efforts in later stages of the EXIT treatment is clearly caused by self-selection of strong types into continuing conflict, rather than some kind of misunderstanding and learning of how to play the game. In addition, discouraging signals obtained about the type distribution cause an increase in the probability to exit.

\subsection{On beliefs and updating}

The theory framework predicts that individual beliefs about the opponent's effort in early stages/rounds should be positively correlated with the own type (effort), while this correlation is reduced once the individuals have obtained sufficiently many signals about other players' types through the observed effort choices. ${ }^{37}$ Figure 2 plots the correlation coefficient of own effort and stated beliefs about the respective opponent's effort over the 15 rounds and the (up to) 5 stages within one round, separately for the sessions with and without monetary incentives for belief elicitation. In both cases, the figure shows a rapid reduction in the correlation in early rounds but then a rather stable positive correlation, suggesting that the own type matters for the beliefs about other players' types even in later stages/rounds. ${ }^{38}$ While the reduction in correlation is in line with the theory predictions, the persistence of considerable correlation is not explained in our theory. This persistence is, however, well in line with considerable psychological evidence on social projection, the confirmation bias, and in this context on the primacy effect in belief formation (see Marks and Miller 1987 on social projection, and Nickerson 1998 on the confirmation bias).

As further evidence on updating of beliefs, Table 4 in Section B.4 of the Online Appendix shows that, similar to the correlation coefficient in Fig. 2, the deviation of stated beliefs from the actual effort of the opponent decreases in the number of signals obtained about others' efforts (the number of the state contest played) but at a decreasing rate. Put differently, the accuracy of the stated beliefs increases rapidly across the first stages and rounds but the learning effects weaken in later stages where the individuals should already have a rather accurate prior. Again, this holds very similarly for the sessions with and without monetary incentives for the subjects for stating correct beliefs. ${ }^{39}$

\footnotetext{
36 The sum of the coefficients of $\mathbf{1}_{x_{-i r 1}>E_{i r 1}\left(x_{-i r 1}\right)}$ and its interaction with the dummy $\mathbf{1}_{\text {late }}$ for later rounds is significant at the $10 \%$-level ( $p$ value is 0.086 ).

37 More precisely, the predicted correlation between an individual's own effort and her expectation of the opponent's effort is equal to one in the first contest played and approaches zero in the limit case where there is common knowledge about the true type distribution. The correlation is supposed to decrease more strongly in early stages where the informational value of an additional signal is higher. In contrast, the benchmark based on complete information (Proposition 1) would predict no correlation.

38 Previous contest experiments have typically found a positive correlation of stated beliefs and own effort; compare Bhattacharya (2016) on symmetric and asymmetric group contests and Sheremeta (2018) for a static two-player contest. Apart from differences in the setup, these papers do not investigate (typedependent) adjustments of beliefs throughout the contests played.

39 As further support for the reliability of the data on beliefs even in the sessions where belief elicitation was not incentivized, Fig. 3 in Section B.5 of the Online Appendix shows the distribution of the deviation of the stated beliefs from the actual opponent efforts for the different session types. In the sessions of the BASE treatment without monetary incentives for correct beliefs, the (absolute value of the) deviation
} 

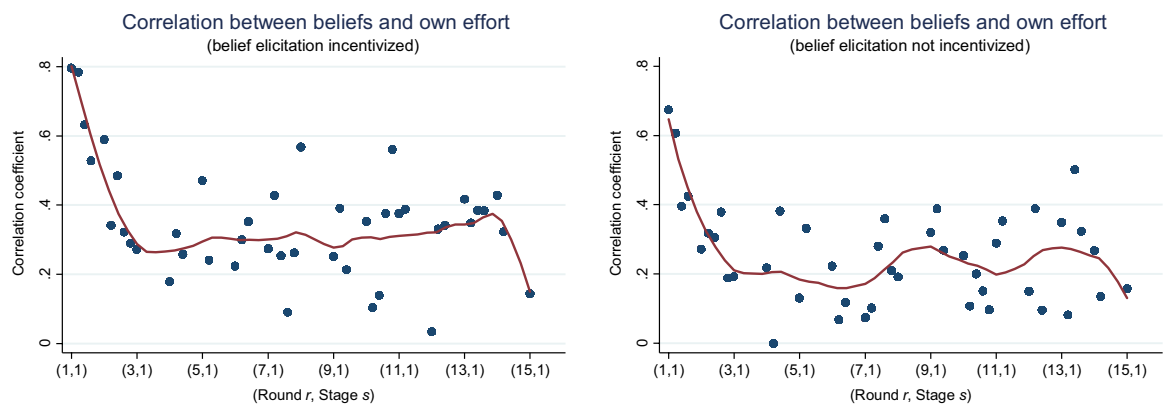

Fig. 2 Correlation between own effort $x_{i r s}$ and stated beliefs $E_{i r s}\left(x_{-i r s}\right)$ about the opponent's effort (in a given stage and round)

Result 4 Individual beliefs about opponents' efforts are positively correlated with the own type. This correlation is reduced in later stages and rounds.

Within one round, different types of players may adjust their beliefs differently. Table 5 in Section B.6 of the Online Appendix presents random-effects regressions where we estimate the stated beliefs as a function of the stage and distinguish between strong and weak types (we basically use the specifications of Table 1, replacing the dependent variable by individual beliefs $E_{\text {irs }}\left(x_{-i r s}\right)$ ). The results confirm the theory predictions in that strong types also hold higher beliefs about the opponent's effort than weak types. This difference is large in early rounds (26.5 points; compare the coefficient of $\mathbf{1}_{\text {strong type }}$ in estimation 2) and becomes smaller in later rounds (estimation 3) where heterogeneity in beliefs is predicted to disappear. Moreover, the adjustment effect of beliefs is more pronounced for strong than for weak types (compare the coefficient of " $\mathbf{1}_{\text {strong type }} \times$ Stage $_{s-1}$ " in estimations $1-3)$, in line with theory prediction in the presence of an empirical type distribution exhibiting many weak types where the updating is predicted to be more important for strong than for weak types. More precisely, in early rounds (estimation 2), both types' beliefs exhibit a large and significant downward adjustment ( $p$ values of the coefficients of "Stage " $_{s-1}$ " and of the sum of the coefficients of "Stage ${ }_{s-1}$ " and its interaction with $\mathbf{1}_{\text {strong type }}<0.001$ ), the adjustment being significantly larger for strong types. In later rounds (estimation 3), weak types' beliefs do not significantly change across stages (see the coefficient of "Stage ${ }_{s-1}$ "; $p$ value is 0.439 ) and the adjustment of strong types' beliefs becomes weaker in size and significance (the sum of the coefficients of "Stage ${ }_{s-1}$ " and its interaction with $\mathbf{1}_{\text {strong type }}$ is significant at the $5 \%$ level; $p$ value is 0.030 ). Finally, the stage 1 opponent's effort has a significantly positive effect on the stated beliefs, especially in early rounds and less so in later rounds (see the coefficient of $x_{-i r 1}$ in estimations 2 and 3; a similar result

Footnote 39 (continued)

of the stated beliefs from the actual opponent effort is on average 39.2, with a median of 21 and a standard deviation of 59.9. In the sessions with monetary incentives for correct beliefs, the average deviation is 42.1 , with a median of 20 and a standard deviation of 63.2. 
is obtained for the previous opponent's effort $\left.x_{-i r, s-1}\right)$. Again, this is in line with the theory mechanism where the individuals update their beliefs from interaction to interaction but at a decreasing rate.

Overall, consistent with the results on individual efforts, the adjustments of beliefs are stronger in early rounds and weakened in late rounds. Also, the difference in average beliefs between strong and weak types is larger in early rounds. Similar adjustment effects in early as compared to late rounds are obtained when estimating strong and weak types' adjustments of beliefs across contest subgames played. This confirms the importance of the own type for belief formation and updating in early stages and rounds.

\section{Conclusions}

This paper studied learning and selection and their implications for possible effort escalation in a simple game of dynamic property rights conflict: a multi-stage contest with random resolve. Players who may differ in unobserved preference characteristics encounter changing adversaries in a sequence of contests of stochastic length. They can make use of what they know about their own type and the actions chosen by their previous adversaries. This way they can learn about the underlying population of players and make inference about the types of current and future adversaries. In a corresponding lab experiment, we find that participants exploit the information about their own type (self-projection) and observations about other players' actions to update their beliefs about future adversaries' effort. Belief updating can explain type-dependent effort escalation and de-escalation, respectively, across the stages of conflict in an otherwise stationary environment. Moreover, whenever there is a possibility to exit the game, effort escalation is caused by self-selection based on preference heterogeneity and perceptions about the conflict environment. Learning in the experiment falls short of perfectly rational Bayesian updating, however, and psychological behavioral theories of confirmation bias and the primacy effect in belief formation over time seemingly play a role.

The paper contributes both to the theory of conflict and to the methodology of conflict experiments and related strategic interactions. From a methodological perspective, it highlights the role of the theoretical benchmark. The benchmark of equilibrium behavior between identical players who maximize their monetary payoffs under conditions of full information about the environment does not account for problems of incomplete information that the subjects face. Unobserved preference heterogeneity in non-monetary payoff components turns laboratory experiments into games under incomplete information, even when the rules of the game and the structure of monetary payoffs are common knowledge. Consequently, the subjects in the laboratory might suffer from uncertainty about the likely composition of types in the population of players they encounter. Their own types are then predictors about this composition; Bayesian updating causes self-projection in this case. Also, players' experience from previous interactions provides information about the types that players face in later interactions so that adjustments in behavior across interactions are a natural consequence of standard Bayesian updating. In other words, with type 
heterogeneity and incomplete information, systematic deviations from the standard theory prediction for symmetric players naturally emerge due to differences in players' beliefs and experience and, whenever possible, due to self-selection of certain player types. From an ex ante perspective (that is, unconditional on the true type distribution), average behavior under the benchmark of symmetric players or complete information may not be qualitatively very different from the average behavior with uncertainty and updating. But since the adjustments in behavior can depend on a player's preference type and the distribution of preference types in the population, this is most likely no longer true ex post (that is, conditional on the true type distribution) and at the level of player types. Therefore, the predictions for behavior in a single experiment can be structurally different when taking into account unobserved preference heterogeneity and uncertainty about the true type distribution. Our experiment suggests that these modified predictions can be a suitable benchmark for testing and interpreting conflict behavior.

Welfare considerations are not straightforward and comprise several relevant and conflicting aspects. Contest effort itself can be desirable or wasteful. Much effort might be appreciated in sports competitions, design contests or R\&D, and wasteful in military conflict or plain property-rights conflict. In some applications even the welfare assessment of effort is ambiguous. Lobbying effort, for instance, might be informative [as in Skaperdas and Vaidya (2012)], potentially leading to better political decisions, or be simply wasteful [as in the standard Tullock (1980) approach]. Similarly, a focus on the participants' payoffs as a measure of welfare leads to ambiguities. We might conclude that an exit option is good for weak players who can enjoy an outside option without fighting a battle against determined fighters. For strong players, an exit option has negative indirect effects at the individual level, as it intensifies the competition between them. But the set of strong players as a whole benefits because exit of weak players allocates the prize among fewer players who value it more. Finally, even though improved information about the population benefits a player when keeping others' behavior constant, there are countervailing effects on the players' payoffs due to the different strategic implications of learning for strong and weak players. For instance, learning that the population mostly consists of weak types helps strong players to avoid excessive expenditures but, at the same time, lowers their chances of winning because weak types become more competitive.

One of the main messages of the paper relates to the role of self-projection as a tool for an assessment of potentially unfamiliar conflict situations that involve population uncertainty. If players do not have a sound basis for assessing the types of their adversaries, then self-projection is seemingly a useful device they apply to improve their strategy choices. But in line with evidence from psychology, what they learn from self-projection might be more persistent than what would be optimal. Without going so far as to make recommendations for possible correction policies, the persistence of self-projection and the lack of sufficiently fast updating of prior beliefs may still be useful as a finding when drawing policy conclusions. 
Acknowledgements Open access funding provided by University of Innsbruck and Medical University of Innsbruck.

Open Access This article is distributed under the terms of the Creative Commons Attribution 4.0 International License (http://creativecommons.org/licenses/by/4.0/), which permits unrestricted use, distribution, and reproduction in any medium, provided you give appropriate credit to the original author(s) and the source, provide a link to the Creative Commons license, and indicate if changes were made.

\section{Appendix 1: proofs of the main theoretical results}

\section{Proof of Proposition 1}

Consider the static contest game in the final stage $n$ if $n$ is reached. All actions that were observed in previous stages do not affect beliefs, as $\pi_{\omega}$ is common knowledge. This continuation game is equivalent to a static game with incomplete information about $v_{i}$. Backward induction yields that this is also true for each previous contest encounter. The dynamic problem decomposes into $n$ independent static equilibrium problems, each with the same (but type-dependent) effort choice. All player types expect the opponent's effort at stage $s$ to be as in (4), based on the commonly known population shares. An interior equilibrium $\left(\breve{x}_{v_{H}}, \breve{x}_{v_{L}}\right) \in(\underline{x}, \bar{x})^{2}$ is described by the first-order conditions ${ }^{40}$

$$
q \pi_{\omega} \frac{\breve{x}_{v_{H}}}{\left(\breve{x}_{v_{i}}+\breve{x}_{v_{H}}\right)^{2}} v_{i}+q\left(1-\pi_{\omega}\right) \frac{\breve{x}_{v_{L}}}{\left(\breve{x}_{v_{i}}+\breve{x}_{v_{L}}\right)^{2}} v_{i}-1=0 \text { for } i \in\{H, L\} .
$$

\section{Proof of Proposition 2}

Suppose that stage $s$ is reached (which implies that the prize has not been awarded in any previous stage) and consider the decision problem of player $i$ who is fully characterized by the vector $\mathbf{h}_{i, s} \equiv\left(v_{i}, x_{-i, 1}, \ldots x_{-i, s-1}\right)$. At stage $s$, a player of type $\mathbf{h}_{i, s}$ forms probability beliefs for all possible types $\mathbf{h}_{j, s} \in H_{s}$. Due to random matching from a set with infinitely many players, these beliefs are also the probability beliefs about the type of the opponent $-i$ at stage $s$. The player also forms expectations about each type $\mathbf{h}_{j, s}$ 's effort choice at stage $s$. Assuming that the effort choices are single-valued we denote these by $x_{\mathbf{h}_{j, s}}$.

Denote a player's expected continuation payoff from stage $s+1$ onward by $V_{s+1}\left(\mathbf{h}_{i, s}\right)$. Then, at stage $s$ player $i$ maximizes the objective function

$$
q \int_{\mathbf{h}_{j, s} \in H_{s}} \frac{x_{\mathbf{h}_{i, s}}}{x_{\mathbf{h}_{i, s}}+x_{\mathbf{h}_{j, s}}} v_{i} d F_{\mathbf{h}_{i, s}}\left(\mathbf{h}_{j, s}\right)-x_{\mathbf{h}_{i, s}}+(1-q) V_{s+1}\left(\mathbf{h}_{i, s}\right)
$$

\footnotetext{
${ }^{40}$ With the minimum effort requirement of $x>0$, the equilibrium is an interior equilibrium unless $v_{H} / v_{L}$ becomes very large (in the latter case, low types exert equilibrium effort of $\underline{x}>0$ ).
} 
subject to $x \in[\underline{x}, \bar{x}]$. As we will argue further below, the continuation values $V_{s+1+k}\left(\mathbf{h}_{i, s}\right)$ for $k=0, \ldots, n-(s+1)$ are exogenous with respect to a single player's choice at stage $s$. We also will show that, for each stage $s, F_{\mathbf{h}_{i, s}}$ consists of finite sets of probability atoms in the candidate equilibrium. We use these properties to rewrite the objective function as

$$
q \sum_{\mathbf{h}_{j, s} \in H_{s}} \rho_{\mathbf{h}_{i, s}}\left(\mathbf{h}_{j, s}\right) \frac{x_{\mathbf{h}_{i, s}}}{x_{\mathbf{h}_{i, s}}+x_{\mathbf{h}_{j, s}}} v_{i}-x_{\mathbf{h}_{i, s}}+(1-q) V_{s+1}\left(\mathbf{h}_{i, s}\right) .
$$

As $x_{\mathbf{h}_{i s}} \geq \underline{x}>0$, the objective function is continuous and strictly concave in $x_{\mathbf{h}_{i s}}$. It must take a unique maximum on the closed and compact support of possible effort choices, and this maximum must be a continuous function of efforts $x_{\mathbf{h}_{j, s}}$. Hence, the optimal choices of the players define a continuous self-mapping

$$
\begin{aligned}
{[\underline{x}, \bar{x}]^{\# H_{s}} } & \rightarrow[\underline{x}, \bar{x}]^{\# H_{s}} \\
\mathbf{x}_{s} & \rightarrow \boldsymbol{\xi}\left(\mathbf{x}_{s}\right)
\end{aligned}
$$

on a convex and compact set, where \# $H_{s}$ is the number of types in stage $s$ emerging from histories in stages $k=1$ to $k=s-1$. Applying Brouwer's fixed point theorem yields that $\xi\left(\mathbf{x}_{s}\right)$ has at least one fixed point, and this fixed point constitutes a vector of equilibrium efforts at stage $s$. Suppose in what follows that if there are multiple fixed points, the players coordinate on one of them.

Note that $H_{1}, \ldots, H_{n}$ is comprised of sets with finite numbers of elements on the equilibrium path. For $s=1$, this set has only two elements, as $\mathbf{h}_{i, 1} \in\left\{v_{H}, v_{L}\right\}$. A given set $H_{s}$ with a finite number of types that have positive probabilities that sum up to one leads to a new set of types $H_{s+1}$ in this equilibrium with the same property: the induced set fulfills all assumptions made about $H_{s}$, and in particular, is a finite set if $H_{s}$ was a finite set. So, should the contest not resolve at stage $s$, it moves on to $s+1$ and the problem at $s+1$ has a larger, but finite number of types and is structurally equivalent to the problem at $s$.

Also, a player's belief that $V_{s+1}\left(\mathbf{h}_{i, s}\right)$ is independent of a single player's action at stage $s$ is correct. If a player of type $\mathbf{h}_{j, s}$ deviates from the local equilibrium strategy profile $\mathbf{x}_{s}^{*}$ at stage $s$ and chooses $x \neq x_{\mathbf{h}_{j, s}}^{*}$, the deviating behavior is observed by the stage $s$ opponent $-j$ and changes the history type of this player $-j$. However, given that the contestants are randomly re-matched at each stage and that player $-j$ and all players that $-j$ is matched with constitute a set with zero measure within the set of all players, the deviating behavior does not change the equilibrium composition of types in the next or any other future stage. Therefore, the deviation has no effect on the player's own payoff in future stages.

Hence, the solution for a perfect Bayesian equilibrium of the game reduces to a series of Bayesian equilibria, one for each possible stage of the game. This sequence of problems is linked only by the fact that the local strategy profile at a given stage together with the equilibrium distribution of types $\mathbf{h}_{i, s} \in H_{s}$ at the beginning of stage $s$ jointly determine the equilibrium distribution of types $\mathbf{h}_{i, s+1} \in H_{s+1}$ at the beginning of stage $s+1$. 


\section{Proof of Lemma 1}

Ex ante, all players have the same common prior about the likelihood of the two states of the world. With the common prior belief which assigns a probability of 1 / 2 to each of the two states, Bayesian updating leads to

$$
\begin{aligned}
\sigma_{v_{H}}(\bar{\omega}) & =\operatorname{prob}\left(\omega=\bar{\omega} \mid v_{i}=v_{H}\right)=\frac{\operatorname{prob}\left(v_{i}=v_{H} \mid \omega=\bar{\omega}\right) \operatorname{prob}(\omega=\bar{\omega})}{\operatorname{prob}\left(v_{i}=v_{H}\right)} \\
& =\frac{\pi_{\bar{\omega}} \frac{1}{2}}{\frac{1}{2} \pi_{\bar{\omega}}+\frac{1}{2} \pi_{\underline{\omega}}}=\frac{1}{2}+d
\end{aligned}
$$

for a player who learns to have a valuation $v_{H}$. This belief induces a belief about the share of strong types in the population and the probability that the stage 1 opponent is of this type. This share/probability is

$$
\rho_{v_{H}}\left(v_{H}\right)=\sigma_{v_{H}}(\bar{\omega}) \pi_{\bar{\omega}}+\left(1-\sigma_{v_{H}}(\bar{\omega})\right) \pi_{\underline{\omega}}=\frac{1}{2}+2 d^{2} .
$$

Analogously, for a player who learns to have a valuation $v_{L}$, Bayesian updating yields

$$
\sigma_{v_{L}}(\bar{\omega})=\operatorname{prob}\left(\omega=\bar{\omega} \mid v_{i}=v_{L}\right)=\frac{\left(1-\pi_{\bar{\omega}}\right) \frac{1}{2}}{\frac{1}{2}\left(1-\pi_{\bar{\omega}}\right)+\frac{1}{2}\left(1-\pi_{\underline{\omega}}\right)}=\frac{1}{2}-d
$$

so that the posterior belief of weak types about the share of strong types is

$$
\rho_{v_{L}}\left(v_{H}\right)=\sigma_{v_{L}}(\bar{\omega}) \pi_{\bar{\omega}}+\left(1-\sigma_{v_{L}}(\bar{\omega})\right) \pi_{\underline{\omega}}=\frac{1}{2}-2 d^{2} .
$$

We note that $\sigma_{v_{H}}(\bar{\omega})>\sigma_{v_{L}}(\bar{\omega})$ and $\rho_{v_{H}}\left(v_{H}\right)>\rho_{v_{L}}\left(v_{H}\right)$.

\section{Proof of Proposition 3}

The stage 1 effort choice of type $\mathbf{h}_{i, 1} \in\left\{v_{H}, v_{L}\right\}$ maximizes

$$
q\left[\rho_{v_{i}}\left(v_{H}\right) \frac{x_{i, 1}}{x_{i, 1}+x_{v_{H}}^{*}} v_{i}+\left(1-\rho_{v_{i}}\left(v_{H}\right)\right) \frac{x_{i, 1}}{x_{i, 1}+x_{v_{L}}^{*}} v_{i}\right]-x_{i, 1}+V_{2}\left(\mathbf{h}_{i, 1}\right) .
$$

Since the continuation payoff $V_{2}\left(\mathbf{h}_{i, 1}\right)$ does not depend on $x_{i, 1}$, the equilibrium efforts $\left(x_{v_{H}}^{*}, x_{v_{L}}^{*}\right)$ in an interior Bayesian Nash equilibrium are the solution of the system of two equations 


$$
\begin{aligned}
& \left(1-\rho_{v_{H}}\left(v_{H}\right)\right) q v_{H} \frac{x_{v_{L}}^{*}}{\left(x_{v_{H}}^{*}+x_{v_{L}}^{*}\right)^{2}}=1-\rho_{v_{H}}\left(v_{H}\right) q v_{H} \frac{1}{4 x_{v_{H}}^{*}} \\
& \rho_{v_{L}}\left(v_{H}\right) q v_{L} \frac{x_{v_{H}}^{*}}{\left(x_{v_{L}}^{*}+x_{v_{H}}^{*}\right)^{2}}=1-\left(1-\rho_{v_{L}}\left(v_{H}\right)\right) q v_{L} \frac{1}{4 x_{v_{L}}^{*}}
\end{aligned}
$$

which are directly obtained from the first-order conditions. Since (23) and (25) imply that $1-\rho_{v_{H}}\left(v_{H}\right)=\rho_{v_{L}}\left(v_{H}\right)$, combining the two equations in (27) yields

$$
\frac{v_{H}}{v_{L}}=\frac{x_{v_{H}}^{*}}{x_{v_{L}}^{*}} .
$$

Note that (28) together with $v_{H}>v_{L}$ implies that $x_{v_{H}}^{*}>x_{v_{L}}^{*}$. With (27) and (28) we obtain the equilibrium values (8) and (9). This characterizes the equilibrium effort choices at stage 1 if $\left(x_{v_{H}}^{*}, x_{v_{L}}^{*}\right) \in(\underline{x}, \bar{x})^{2}$. A sufficient condition for the equilibrium to be interior is that $\bar{x} \geq q v_{H}$ and $\underline{x}$ is sufficiently close to zero.

Each player $i$ anticipates the equilibrium effort levels (8) and (9) of their matched opponent $-i$, and these anticipated values are independent of $i$ 's own type. But the players' posterior beliefs about their opponent's type depend on the own type, as given in (23) and (25). Player $i$ uses this posterior belief to calculate the unconditional expected effort of $-i$ as (10) and (11). Due to $\rho_{v_{H}}\left(v_{H}\right)>\rho_{v_{L}}\left(v_{H}\right)$ and $x_{v_{H}}^{*}>x_{v_{L}}^{*}$, we get $E_{v_{H}}\left(x_{-i}\right)>E_{v_{L}}\left(x_{-i}\right)$.

\section{Proof of Proposition 4}

Combining the two first-order conditions (5) and (6) yields the condition

$$
\frac{\breve{x}_{v_{L}}}{\frac{q v_{L}}{4}}\left(1-\pi_{\omega}\right)=\frac{\breve{x}_{v_{H}}}{\frac{q v_{H}}{4}} \pi_{\omega}+\left(1-2 \pi_{\omega}\right),
$$

which can be used to establish monotonicity properties of the equilibrium efforts: $\breve{x}_{v_{H}}$ is strictly increasing in $\pi_{\omega}$ and $\breve{x}_{v_{L}}$ is strictly decreasing in $\pi_{\omega}{ }^{41}$ Moreover, $\breve{x}_{v_{H}}$ and $\breve{x}_{v_{L}}$ are continuous is $\pi_{\omega}$, that is, continuous in $d$, and stage 1 equilibrium efforts $x_{v_{H}}^{*}$ and $x_{v_{L}}^{*}$ are continuous in $d$ as well. Using (8) and (9),

$$
\lim _{d \rightarrow 0} x_{v_{H}}^{*}=\frac{1}{2} \frac{q v_{H}}{4}+\frac{1}{2} \frac{q v_{H}^{2} v_{L}}{\left(v_{H}+v_{L}\right)^{2}}=\lim _{d \rightarrow 0} \breve{x}_{v_{H}}
$$

and

\footnotetext{
41 This monotonicity result follows as in Serena (2018, Proposition 5); therefore, a proof is omitted. Intuitively, equilibrium efforts are increasing in the probability of facing a type with the same valuation.
} 


$$
\lim _{d \rightarrow 0} x_{v_{L}}^{*}=\frac{1}{2} \frac{q v_{L}}{4}+\frac{1}{2} \frac{q v_{H} v_{L}^{2}}{\left(v_{H}+v_{L}\right)^{2}}=\lim _{d \rightarrow 0} \breve{x}_{v_{L}}
$$

in both states $\omega \in\{\bar{\omega}, \underline{\omega}\}^{42}$

Part (ii) Suppose that the true share of high-valuation types is $\pi_{\omega}$. Then, $\rho_{v_{H}}\left(v_{H}\right)>1 / 2>\pi_{\underline{\omega}}$ for all $d \in(0,1 / 2)$. Using (30) and the fact that $x_{v_{H}}^{*} \frac{\pi^{\omega}}{\bar{i}}$ strictly increasing in $\rho_{v_{H}}\left(v_{H}\right)$ (Corollary 1) and $\breve{x}_{v_{H}}$ is strictly increasing in $\pi_{\omega}$, it follows that $\breve{x}_{v_{H}}<x_{v_{H}}^{*}$ for all $d \in(0,1 / 2)$. Note that $\breve{x}_{v_{H}}\left(\pi_{\underline{\omega}}\right)$ is lowest if $d \rightarrow 1 / 2$ in which case $\breve{x}_{v_{H}}$ converges to

$$
\frac{q v_{H}^{2} v_{L}}{\left(v_{H}+v_{L}\right)^{2}}=\frac{q v_{L}}{4} \frac{\left(v_{H}+v_{H}\right)^{2}}{\left(v_{H}+v_{L}\right)^{2}}>\frac{q v_{L}}{4}
$$

hence, for all $d \in(0,1 / 2), \breve{x}_{v_{H}}\left(\pi_{\underline{\omega}}\right)$ is higher than the stage 1 effort $x_{v_{L}}^{*}$ of weak types (which is at most $q v_{L} / 4$, in case $\bar{\rho}_{v_{L}}\left(v_{L}\right) \rightarrow 1$ ).

Now consider the low-valuation types. With the first-order condition for $x_{v_{L}}^{*}$,

$$
\begin{aligned}
0 & =q \rho_{v_{L}}\left(v_{H}\right) \frac{x_{v_{H}}^{*}}{\left(x_{v_{L}}^{*}+x_{v_{H}}^{*}\right)^{2}} v_{L}+q\left(1-\rho_{v_{L}}\left(v_{H}\right)\right) \frac{x_{v_{L}}^{*}}{\left(x_{v_{L}}^{*}+x_{v_{L}}^{*}\right)^{2}} v_{L}-1 \\
& <q \pi_{\underline{\omega}} \frac{x_{v_{H}}^{*}}{\left(x_{v_{L}}^{*}+x_{v_{H}}^{*}\right)^{2}} v_{L}+q\left(1-\pi_{\underline{\omega}}\right) \frac{x_{v_{L}}^{*}}{\left(x_{v_{L}}^{*}+x_{v_{L}}^{*}\right)^{2}} v_{L}-1 \\
& <\pi_{\underline{\omega}} \frac{\breve{x}_{v_{H}}}{\left(x_{v_{L}}^{*}+\breve{x}_{v_{H}}\right)^{2}} v_{L}+q\left(1-\pi_{\underline{\omega}}\right) \frac{x_{v_{L}}^{*}}{\left(x_{v_{L}}^{*}+x_{v_{L}}^{*}\right)^{2}} v_{L}-1 .
\end{aligned}
$$

Here, the first inequality follows from $\pi_{\underline{\omega}}<\rho_{v_{L}}\left(v_{H}\right)$; the second inequality follows from $\breve{x}_{v_{H}}<x_{v_{H}}^{*}{ }^{43}$ Let $d \in(0,1 / 2)$ and suppose that $\breve{x}_{v_{L}} \leq x_{v_{L}}^{*}$. Then, the right-hand side of inequality (33) is (weakly) smaller than

$$
q \pi_{\underline{\omega}} \frac{\breve{x}_{v_{H}}}{\left(\breve{x}_{v_{L}}+\breve{x}_{v_{H}}\right)^{2}} v_{L}+q\left(1-\pi_{\underline{\omega}}\right) \frac{\breve{x}_{v_{L}}}{\left(\breve{x}_{v_{L}}+\breve{x}_{v_{L}}\right)^{2}} v_{L}-1,
$$

\footnotetext{
42 If $d \rightarrow 0$ then $\lim _{d \rightarrow 0} \pi_{\bar{\omega}}=\lim _{d \rightarrow 0} \pi_{\underline{\omega}}=\lim _{d \rightarrow 0} \rho_{v_{H}}\left(v_{H}\right)=\lim _{d \rightarrow 0} \rho_{v_{L}}\left(v_{H}\right)=1 / 2$.

${ }^{43}$ The marginal probability $\partial p_{i}\left(x_{i}, x_{-i}\right) / \partial x_{i}=x_{-i} /\left(x_{i}+x_{-i}\right)^{2}$ is strictly increasing for all $x_{-i}<x_{i}$, reaches its maximum at $x_{-i}=x_{i}$, and is strictly decreasing for $x_{-i}>x_{i}$. Therefore, since $x_{v_{L}}^{*}<\breve{x}_{v_{H}}<x_{v_{H}}^{*}$, we get $x_{v_{L}}^{*} /\left(x_{v_{L}}^{*}+x_{v_{L}}^{*}\right)^{2}>\breve{x}_{v_{H}} /\left(x_{v_{L}}^{*}+\breve{x}_{v_{H}}\right)^{2}>x_{v_{H}}^{*} /\left(x_{v_{L}}^{*}+x_{v_{H}}^{*}\right)^{2}$ (the ranking $x_{v_{L}}^{*}<\breve{x}_{v_{H}}$ holds as $x_{v_{L}}^{*}<q v_{L} / 4<\breve{x}_{v_{H}}$ for all $d \in(0,1 / 2)$; see above) .
} 
which is equal to zero by (18); contradiction. Therefore, we must have $\breve{x}_{v_{L}}>x_{v_{L}}^{*}$. If $d \rightarrow 1 / 2$, the probability that weak types attach to facing another weak type converges to one so that both $x_{v_{L}}^{*}$ and $\breve{x}_{v_{L}}$ converge to $q v_{L} / 4$.

Part (i) Suppose that the true share of high-valuation types is $\pi_{\bar{\omega}}$. Then, $\rho_{v_{L}}\left(v_{L}\right)>1 / 2>1-\pi_{\bar{\omega}}$ for all $d \in(0,1 / 2)$. Using (31) and the fact that $x_{v_{L}}^{*}$ is strictly increasing in $\rho_{v_{L}}\left(v_{L}\right)$ (Corollary 1 ) and $\breve{x}_{v_{L}}$ is strictly increasing in $1-\pi_{\bar{\omega}}$, it holds that $\breve{x}_{v_{L}}<x_{v_{L}}^{*}$.

Note that the proof of Proposition 4 only makes use of the rankings $\rho_{v_{L}}\left(v_{H}\right)<\pi_{\bar{\omega}}$ as well as $\rho_{v_{H}}\left(v_{H}\right)>\pi_{\omega}$ and $\rho_{v_{L}}\left(v_{H}\right)>\pi_{\underline{\omega}}$, but does not use, for instance, that $\rho_{v_{L}}\left(v_{H}\right)=1 / 2-2 d^{2}$ and $\pi_{\underline{\omega}}=1 / 2-d$ for the assumed information structure. In other words, the result in Proposition 4 does not qualitatively depend on the players knowing the exact share of high and low players in the population. It is sufficient that the players hold common beliefs about the type distribution and that the beliefs about the share of high types are corrected upward in case of $\omega=\bar{\omega}$, and are corrected downward in case of $\omega=\omega$.

In contrast, in case of $\omega=\bar{\omega}$, comparisons of $\breve{x}_{v_{H}}\left(\pi_{\bar{\omega}}\right)$ to $x_{v_{H}}^{*}$ rely on the difference between $\pi_{\bar{\omega}}$ and $\rho_{v_{H}}\left(v_{H}\right)$. It is possible (although more complex) to show that $\breve{x}_{v_{H}}\left(\pi_{\bar{\omega}}\right)>x_{v_{H}}^{*}$ for all $\left(v_{H}, v_{L}\right)$ with $v_{H}>v_{L}$ if $d$ is sufficiently small. However, $\breve{x}_{v_{H}}$ may be smaller than $x_{v_{H}^{*}}^{*}$ if $d$ is very close to $1 / 2$ and $v_{H}$ is large compared to $v_{L}{ }^{44}$

\section{Proof of Proposition 5}

Suppose all players $i$ with $v_{i}=v_{L}$ exit and all players $j$ with $v_{j}=v_{H}$ continue in stage 2 . We have to show that no single player has an incentive to deviate from the candidate equilibrium behavior. In the candidate equilibrium, the players anticipate that the common beliefs among all players is that (almost) all players who remain active in stage 2 have $v_{j}=v_{H}$. Hence, players with $v_{j}=v_{H}$ choose an equilibrium effort of $x_{j, s}=q v_{H} / 4$ in all stages $s=2, \ldots, n$ and earn an expected payoff from staying active of

$$
b_{H} \equiv \frac{q v_{H}}{4} \sum_{k=2}^{k=n}(1-q)^{k-2} \text {. }
$$

A player $i$ with valuation $v_{i}=v_{L}$ can also anticipate that $x_{-i, s}=q v_{H} / 4$ for all $s=2, \ldots n$ and chooses her effort $x_{i, s} \in[\underline{x}, \bar{x}]$ as the best reply. Straightforward calculus yields this best reply as $\max \left\{\tilde{x}_{L}, \underline{x}\right\}$ with

$$
\tilde{x}_{L} \equiv \frac{q}{4}\left(-v_{H}+2 \sqrt{v_{L} v_{H}}\right)
$$

\footnotetext{
$\overline{{ }^{44} \text { For instance, if the true state is } \omega=\bar{\omega} \text { and } q v_{H}=390, q v_{L}}=100$ and $d=0.45$, we obtain $\breve{x}_{v_{H}}=94.091<94.256=x_{v_{H}}^{*}$ and $\breve{x}_{v_{L}}=8.3925<24.168=x_{v_{L}}^{*}$.
} 
at all stages $s=2, \ldots, n$. Denote the resulting continuation value for weak types by

$$
b_{L} \equiv\left(q \frac{\max \left\{\tilde{x}_{L}, \underline{x}\right\}}{\max \left\{\tilde{x}_{L}, \underline{x}\right\}+\frac{q v_{H}}{4}} v_{L}-\max \left\{\tilde{x}_{L}, \underline{x}\right\}\right) \sum_{k=2}^{k=n}(1-q)^{k-2} .
$$

Hence, it is optimal for weak types to exit and for strong types to stay if $b \in\left[b_{L}, b_{H}\right]$.

Note that the interval $\left[b_{L}, b_{H}\right]$ is non-empty. If $\tilde{x}_{L} \geq \underline{x}$ then $b_{L}$ simplifies to

$$
\frac{q}{4}\left(v_{H}-4\left(\sqrt{v_{L} v_{H}}-v_{L}\right)\right) \sum_{k=2}^{k=n}(1-q)^{k-2}
$$

which is strictly smaller than $b_{H}$ given above. If instead $\tilde{x}_{L}<\underline{x}$ then $b_{L}$ is equal to

$$
\left(q \frac{\underline{x}}{\underline{x}+\frac{q v_{H}}{4}} v_{L}-\underline{x}\right) \sum_{k=2}^{k=n}(1-q)^{k-2}<\left(q \frac{\underline{x}}{\underline{x}+\frac{q v_{H}}{4}} v_{H}-\underline{x}\right) \sum_{k=2}^{k=n}(1-q)^{k-2}<b_{H},
$$

where the second inequality holds whenever strong types strictly prefer an effort $q v_{H} / 4$ over an effort $\underline{x}$.

\section{References}

Baharad, E., \& Nitzan, S. (2008). Contest efforts in light of behavioral considerations. Economic Journal, 118(533), 2047-2059.

Baye, M. R., \& Hoppe, H. C. (2003). The strategic equivalence of rent-seeking, innovation, and patentrace games. Games and Economic Behavior, 44(2), 217-226.

Baye, M. R., Kovenock, D., \& de Vries, C. G. (1993). Rigging the lobbying process: An application of the all-pay auction. American Economic Review, 83(1), 289-294.

Ben-Bassat, A. (2011). Conflicts, interest groups, and politics in structural reforms. Journal of Law \& Economics, 54(4), 937-952.

Bhattacharya, P. (2016). Inter-team contests with power differential. Journal of Economic Behavior \& Organization, 132, 157-175.

Brookins, P., \& Ryvkin, D. (2014). An experimental study of bidding in contests of incomplete information. Experimental Economics, 17(2), 245-261.

Camerer, C., \& Weigelt, K. (1988). Experimental tests of a sequential equilibrium reputation model. Econometrica, 56(1), 1-36.

Che, Y.-K., \& Gale, I. (2003). Optimal design of research contests. American Economic Review, 93(3), 646-671.

Clark, D. J., \& Riis, C. (1998). Contest success functions: An extension. Economic Theory, 11(1), 201-204.

Dechenaux, E., Kovenock, D., \& Sheremeta, R. (2015). A survey of experimental research on contests, all-pay auctions and tournaments. Experimental Economics, 18(4), 609-669.

Deck, C., \& Sheremeta, R. M. (2012). Fight or flight? Defending against sequential attacks in the game of siege. Journal of Conflict Resolution, 56(6), 1069-1088.

Deck, C., \& Sheremeta, R. M. (2019). Tug-of-war in the laboratory. European Journal of Political Economy. https://doi.org/10.1016/j.ejpoleco.2019.02.006.

Einy, E., Haimankoa, O., Moreno, D., Sela, A., \& Shitovitz, B. (2015). Equilibrium existence in Tullock contests with incomplete information. Journal of Mathematical Economics, 61, 241-245.

Ellingsen, T. (1991). Strategic buyers and the social cost of monopoly. American Economic Review, 81(3), 648-657. 
Fallucchi, F., Renner, E., \& Sefton, M. (2013). Information feedback and contest structure in rent-seeking games. European Economic Review, 64, 223-240.

Fey, M. (2008). Rent-seeking contests with incomplete information. Public Choice, 135(3-4), 225-236.

Fischbacher, U. (2007). z-Tree: Zurich toolbox for ready-made economic experiments. Experimental Economics, 10(2), 171-178.

Fu, Q., Gürtler, O., \& Münster, J. (2013). Communication and commitment in contests. Journal of Economic Behavior \& Organization, 95, 1-19.

Fudenberg, D., \& Levine, D. K. (1997). Measuring players' losses in experimental games. Quarterly Journal of Economics, 112, 507-536.

Fudenberg, D., \& Takahashi, S. (2011). Heterogeneous beliefs and local information in stochastic fictitious play. Games and Economic Behavior, 71, 100-120.

Fullerton, R. L., \& McAfee, R. P. (1999). Auctioning entry into tournaments. Journal of Political Economy, 107(3), 573-605.

Garfinkel, M. R., \& Skaperdas, S. (2012). Oxford handbook of the economics of peace and conflict. Oxford: Oxford University Press.

Gauriot, R., \& Page, L. (2019). Does success breed success? A quasi-experiment on strategic momentum in dynamic contests. Economic Journal. https://doi.org/10.1093/ej/uez040.

Gelder, A., \& Kovenock, D. (2017). Dynamic behavior and player types in majoritarian multi-battle contests. Games and Economic Behavior, 104, 444-455.

Greiner, B. (2004). An online recruitment system for economic experiments. In K. Kremer \& V. Macho (Eds.), Forschung und wissenschaftliches Rechnen 2003 (pp. 79-93). Göttingen: GWDG Bericht 63, Göttingen Ges. fü r Wiss. Datenverarbeitung.

Harris, C., \& Vickers, J. (1987). Racing with uncertainty. Review of Economic Studies, 54(1), 1-21.

Herbst, L. (2016). Who pays to win again? The joy of winning in contest experiments, Working paper of the Max Planck Institute for Tax Law and Public finance no. 2016-6.

Herbst, L., Konrad, K. A., \& Morath, F. (2015). Endogenous group formation in experimental contests. European Economic Review, 74, 163-189.

Hirshleifer, J., \& Riley, J. G. (1992). The analytics of uncertainty and information. Cambridge: Cambridge University Press.

Jia, H. (2008). A stochastic derivation of the ratio form of contest success functions. Public Choice, 135(3-4), 125-130.

Kawada, C. L. K., Gollwitzer, P. M., Oettingen, G., \& Bargh, J. A. (2004). The projection of implicit and explicit goals. Journal of Personality and Social Psychology, 86(4), 545-559.

Kerschbamer, R., Sutter, M., \& Dulleck, U. (2017). How social preferences shape incentives in (experimental) markets for credence goods. Economic Journal, 127(600), 393-416.

Klumpp, T., \& Polborn, M. K. (2006). Primaries and the new hampshire effect. Journal of Public Economics, 90(6-7), 1073-1114.

Konrad, K. A. (2009). Strategy and dynamics in contests. Oxford: Oxford University Press.

Konrad, K. A., Morath, F., \& Mueller, W. (2014). Taxation and market power. Canadian Journal of Economics, 47(1), 173-202.

Krispenz, A., Dickhäuser, O., \& Reinhard, M.-A. (2016). Assessing task difficulty for other people: When deeper evaluation means “it's more about me!”. Social Psychology of Education, 19(4), 865-877.

Lee, T., \& Wilde, L. L. (1980). Market structure and innovation: A reformulation. Quarterly Journal of Economics, 94(2), 429-436.

Loury, G. C. (1979). Market structure and innovation. Quarterly Journal of Economics, 93(3), 395-410.

Mago, S. D., Samak, A. C., \& Sheremeta, R. M. (2016). Facing your opponents: Social identification and information feedback in contests. Journal of Conflict Resolution, 60(3), 459-481.

Mago, S. D., Sheremeta, R. M., \& Yates, A. (2013). Best-of-three contest experiments: Strategic versus psychological momentum. International Journal of Industrial Organization, 31(3), 287-296.

Malueg, D. A., \& Yates, A. J. (2004). Rent seeking with private values. Public Choice, 119(1-2), 161-178.

Marks, G., \& Miller, N. (1987). Ten years of research on the false-consensus effect: An empirical and theoretical review. Psychological Bulletin, 102(1), 72-90.

Meirowitz, A. (2008). Electoral contests, incumbency advantages, and campaign finance. Journal of Politics, 70(3), 681-699.

Münster, J. (2009). Repeated contests with asymmetric information. Journal of Public Economic Theory, 11(1), 89-118. 
Nickerson, R. S. (1998). Confirmation bias: A ubiquitous phenomenon in many guises. Review of General Psychology, 2(2), 175-220.

Prelec, D. (1998). The probability weighting function. Econometrica, 66(3), 497-527.

Quiggin, J. (1982). A theory of anticipated utility. Journal of Economic Behavior and Organization, 3(4), 324-343.

Ryvkin, D. (2010). Contests with private costs beyond two players. European Journal of Political Economy, 26(4), 558-567.

Serena, M. (2018). Harnessing beliefs to stimulate efforts. SSRN: https://ssrn.com/abstract=2686543. Accessed 16 Aug 2019.

Sheremeta, R. M. (2013). Overbidding and heterogeneous behavior in contest experiments. Journal of Economic Surveys, 27(3), 491-514.

Sheremeta, R. M. (2018). Impulsive behavior in competition: Testing theories of overbidding in rentseeking contests. SSRN. https://ssrn.com/abstract=2676419. Accessed 16 Aug 2019.

Skaperdas, S. (1996). Contest success functions. Economic Theory, 7(2), 283-290.

Skaperdas, S., \& Vaidya, S. (2012). Persuasion as a contest. Economic Theory, 51(2), 465-486.

Tullock, G. (1980). Efficient rent-seeking. In J. Buchanan, et al. (Eds.), Toward a theory of the rentseeking society. College Station: Texas A\&M University Press.

Wärneryd, K. (2012). Multi-player contests with asymmetric information. Economic Theory, 51(2), 277-287.

Wasser, C. (2013a). A note on Bayesian Nash equilibria in imperfectly discriminating contests. Mathematical Social Sciences, 66(2), 180-182.

Wasser, C. (2013b). Incomplete information in rent-seeking contests. Economic Theory, 53(1), 239-268.

Yaari, M. E. (1987). The dual theory of choice under risk. Econometrica, 55(1), 95-115.

Publisher's Note Springer Nature remains neutral with regard to jurisdictional claims in published maps and institutional affiliations. 